\title{
Architecture of the large subunit of the mammalian mitochondrial ribosome
}

\section{Journal Article}

\section{Author(s):}

Greber, Basil J.; Boehringer, Daniel; Leitner, Alexander; Bieri, Philipp; Voigts-Hoffmann, Felix; Erzberger, Jan P.; Leibundgut, Marc; Aebersold, Ruedi; Ban, Nenad

Publication date:

2014-01-23

\section{Permanent link:}

https://doi.org/10.3929/ethz-b-000080459

Rights / license:

In Copyright - Non-Commercial Use Permitted

Originally published in:

Nature 505(7484), https://doi.org/10.1038/nature12890 
Title: Architecture of the Large Subunit of the Mammalian Mitochondrial Ribosome

Authors: Basil J. Greber ${ }^{1,{ }^{*}}$, Daniel Boehringer ${ }^{1,{ }^{*}}$, Alexander Leitner ${ }^{2}$, Philipp Bieri $^{1}$, Felix Voigts-Hoffmann ${ }^{1}$, Jan P. Erzberger ${ }^{1}$, Marc Leibundgut ${ }^{1}$, Ruedi Aebersold ${ }^{2,3, \dagger}$, and Nenad Ban ${ }^{1, \dagger}$

Affiliations: ${ }^{1}$ Department of Biology, Institute of Molecular Biology and Biophysics, Schafmattstrasse 20, ETH Zurich, CH-8093 Zurich, Switzerland ${ }^{2}$ Department of Biology, Institute of Molecular Systems Biology, WolfgangPauli-Strasse 16, ETH Zurich, CH-8093 Zurich, Switzerland ${ }^{3}$ Faculty of Science, University of Zurich, Zurich, Switzerland † Correspondence to: ban@mol.biol.ethz.ch (N.B.); aebersold@imsb.biol.ethz.ch (R.A.)

* These authors contributed equally to this work. 
Abstract: Mitochondrial ribosomes synthesize a number of highly hydrophobic proteins encoded on the genome of mitochondria, the organelles in eukaryotic cells that are responsible for energy conversion by oxidative phosphorylation. The ribosomes in mammalian mitochondria have undergone massive structural changes throughout their evolution, including rRNA shortening and acquisition of mitochondrial-specific ribosomal proteins. Here, we present the three-dimensional structure of the 39S large subunit of the porcine mitochondrial ribosome determined by cryo-electron microscopy at $4.9 \AA$ resolution. The structure, combined with data from chemical crosslinking and mass spectrometry experiments, reveals the unique features of the 395 subunit at near atomic resolution and provides detailed insight into the architecture of the polypeptide exit site. This region of the mitochondrial ribosome has been dramatically remodeled, providing a specialized platform for the synthesis and membrane insertion of the highly hydrophobic protein components of the respiratory chain. 
Main Text: Mitochondrial ribosomes (mitoribosomes) are responsible for protein synthesis in mitochondria. These organelles of endosymbiotic origin ${ }^{1}$ are required for energy conversion by aerobic respiration in eukaryotic cells. Mitoribosomes are more closely related to bacterial ribosomes than to eukaryotic cytosolic ribosomes ${ }^{2}$. However, the mammalian mitoribosome has been strongly altered by acquisition of mitochondrial-specific ribosomal proteins and protein extensions ${ }^{2-5}$, as well as the shortening of the mitochondrial ribosomal RNA (rRNA) ${ }^{6}$. The large 395 subunit of the mammalian mitoribosome catalyzes peptide bond formation during protein synthesis and harbors the nascent polypeptide exit tunnel. The structural evolution of the mammalian mitoribosome was accompanied by a strong functional specialization towards the synthesis of the highly hydrophobic mitochondrial inner membrane proteins ${ }^{7}$. The region around the polypeptide tunnel exit of the mitoribosome serves as a specialized platform for membrane insertion and assembly of these critical mitochondrially-encoded respiratory chain components ${ }^{7-11}$. Defects of the mitochondrial translation system are causally involved in a range of human diseases ${ }^{12}$.

While cryo-electron microscopy (cryo-EM) reconstructions of the bovine mitoribosome at $13.5 \AA^{13}$ and $12.1 \AA^{14}$ resolution have visualized large structural differences compared to the bacterial ribosome, detailed molecular insight into the architecture and arrangement of unique protein and rRNA elements of the mammalian mitoribosome is currently lacking. We have used cryo-EM combined with chemical crosslinking followed by mass spectrometry (CX-MS) to determine the structure of the large subunit of the mammalian 
mitoribosome, providing insight into its overall structure and into the molecular architecture of the polypeptide exit site in particular.

\section{Structure determination}

To obtain structural insights into the mammalian mitochondrial ribosome, we purified mitoribosomes and their subunits from porcine (Sus scrofa) liver and collected cryo-EM data of the 395 mitoribosomal subunit. The cryo-EM reconstruction of the $39 \mathrm{~S}$ ribosomal subunit (Fig. 1) at $4.9 \AA$ resolution (Extended Data Fig. 1) extends the insights obtained from previous reconstructions ${ }^{13}$, revealing the secondary structure elements of the mitoribosomal-specific proteins and protein extensions that form a shell around the conserved core of ribosomal proteins and rRNA (Fig. 1 a, b;

Extended Data Fig. 2). The mitochondrial-specific protein elements are mostly located on the solvent-exposed side of the 39S subunit, forming an extensive network of protein-protein contacts, while the subunit interface side is comparably better conserved (Fig. $\mathbf{1} \mathbf{a}, \mathbf{b}$ ), as observed previously in eukaryotic cytosolic ribosomes ${ }^{15}$. Because reliable de novo tracing of protein chains is not possible at the resolution of the cryo-EM map reported here, we subjected the S. scrofa $39 S$ subunit and the Bos taurus 55 S mitoribosome to CX-MS ${ }^{16}$ to obtain protein-protein crosslinking data, enabling a molecular interpretation of our cryo-EM map (Fig. 1 c). 


\section{Structure of the 16S rRNA}

The density for the mitoribosomal 16S rRNA was clearly identifiable in the 4.9 A cryo-EM map, with separation of individual strands and features that correspond to phosphate positions in well-ordered regions of the structure. Guided by the structure of the 23S rRNA of Thermus thermophilus ${ }^{17}$ we were able to build a three dimensional model of the strongly reduced 16S rRNA, in agreement with previous reports ${ }^{13,14}$, but at a considerably higher level of detail (Fig. 2 a, b, Extended Data Fig. 3, Extended Data Fig. 4).

These reductions are most pronounced in domain I, where helices $\mathrm{H} 7, \mathrm{H} 16$, $\mathrm{H} 18, \mathrm{H} 19$, and $\mathrm{H} 20$ of the 23S rRNA are missing in the 16S rRNA. The resulting groove on the surface of the 395 subunit passes near the polypeptide tunnel exit, creating a lateral opening of the tunnel referred to as the polypeptide accessible site (PAS) ${ }^{13}$. Additional losses of rRNA elements near the polypeptide tunnel exit occur in 16S rRNA domain III.

Although $16 \mathrm{~S}$ rRNA is considered to be the only rRNA component of the 39S subunit of the mitoribosome ${ }^{11}$, we observe additional RNA density at the central protuberance that cannot be accounted for by the 16S rRNA (Fig. 2 c, d). This rRNA density is located in the immediate vicinity of MRPL18 and resembles the structural features of domain $\beta$ of the bacterial $5 S$ rRNA, which is located in the same area and interacts with the bacterial homolog of MRPL18 (Fig. 2 d, Extended Data Fig. 5). However, the observed RNA density is insufficient to account for the entire 5S rRNA, and does not form a 
contact to the main body of the 395 subunit as it is the case for the 5 S rRNA in bacterial and eukaryotic cytosolic ribosomes (Extended Data Fig. 5 e, f). Taken together, our density clearly shows the presence of a second molecule of rRNA in the mammalian mitochondrial ribosome, possibly a portion of the $5 S$ rRNA imported from the cytosol ${ }^{18}$, or alternatively a different RNA species acting as a functional replacement for domain $\beta$ of the 5 S rRNA.

Novel proteins in the cryo-EM map of the 395 subunit

To analyze the protein component of the porcine 39S subunit, we first positioned homology models ${ }^{19}$ of all mitoribosomal homologs of bacterial ribosomal proteins ${ }^{2,5}$ visible in the map (Supplementary Table 1), leaving, however, considerable regions of density unexplained (Fig. 1). Based on visual inspection of the cryo-EM map and aided by the crosslinking data from our mass spectrometry experiments (Fig. 3, Supplementary Table 2), we were able to position homology models of several mitochondrial-specific proteins (Extended Data Fig. 6, Supplementary Table 1). The mitochondrial-specific ribosomal proteins MRPL39, MRPL44, and MRPL45 are localized at the polypeptide tunnel exit, and MRPL38, MRPL52, MRPL49, and ICT1 (also termed MRPL58) are located at the central protuberance.

\section{Architecture of the central protuberance}

The overall appearance of the mitoribosomal central protuberance $(\mathrm{CP})$ is considerably different compared to its bacterial counterpart, featuring rod-like 
densities on each side ${ }^{13}$ that can now be identified as $\alpha$-helices. One of these helices emanates from the CP in the immediate vicinity of the location we assigned to MRPL38 (Fig. 4 a, Extended Data Fig. 6). Strikingly, our CX-MS data show the presence of several crosslinks of MRPL38 to MRPL52, a protein containing a very long predicted $\alpha$-helix, thus establishing the identity of this helix as MRPL52 and confirming the assignment of MRPL38 (Fig. 4 a, Extended Data Fig. 7, Supplementary Table 3). MRPL52 therefore likely stabilizes the mitoribosomal CP by forming contacts to both the body of the 395 subunit and MRPL38 at the top of the CP, possibly compensating for the loss of the 5S rRNA contact to the subunit body (Extended Data Fig. 5 e, f).

ICT1 is a functional peptidyl-tRNA hydrolase that has been stably incorporated into the mitoribosomal large subunit ${ }^{20}$. The structure of ICT1 ${ }^{21}$ reveals that it is homologous to canonical bacterial class I release factors and the ribosomal rescue factor YaeJ, which bind to the ribosomal A-site ${ }^{22,23}$. In our CX-MS experiments, we observe several crosslinks between ICT1 and protein components of the mitoribosomal CP (Supplementary Table 3 and Fig. 3). Furthermore, homology models of ICT1 and one of its crosslinking partners, MRPL49, can be fitted into the density next to each other, localizing ICT1 adjacent to MRPL38 and MRPL49 at the base of the CP, respectively (Fig. 4 b, Extended Data Fig. 6). This position of ICT1 differs from the positions of homologous domains of class I release factors in catalytically activated complexes, where they occupy the ribosomal A-site ${ }^{22,23}$. Consequently, ICT1 bound near MRPL38 cannot directly access the nascent chain to induce its release from a peptidyl-tRNA bound in the P-site. However, 
a permanent position in the A-site is not possible for ICT1 since this would impede translation. Therefore, ICT1 may have a purely architectural role in the 395 subunit, while its described activity in polypeptide release ${ }^{20}$ may be performed by a pool of freely diffusing ICT1, or alternatively, polypeptide release by ICT1 may require large-scale displacements of either the P-site tRNA or ICT1 itself.

\section{Architecture of the tunnel exit region}

At the polypeptide exit site, density corresponding to the core folds of the mitoribosomal homologs of bacterial L23 (MRPL23), L29 (MRPL47 ${ }^{5}$ ), L22 (MRPL22), L24 (MRPL24), and L17 (MRPL17) indicates that the ring of proteins surrounding the polypeptide exit site is conserved (Extended Data Fig. 8). However, the tunnel exit region of the mammalian 395 subunit also shows a large number of mitochondrial-specific features, including the previously described PAS ${ }^{13}$, as well as a second layer of protein density, which we were able to partially interpret by docking homology models of the mitoribosomal-specific proteins MRPL39, MRPL44, and MRPL45 (Fig. 4 c, d and Extended Data Fig. 6). Their localization is consistent with the results of our CX-MS experiments (Extended Data Fig. 9 and Supplementary Table 3). MRPL39 and MRPL45 form a second layer of protein on top of the conserved proteins that surround the bacterial polypeptide tunnel exit. MRPL39 is located in close proximity of MRPL22, and MRPL45 is bound to MRPL22 and MRPL24 (Extended Data Fig. 8 c). Both MRPL39 and MRPL44 associate with the 395 subunit via protein-protein interactions, but show 
homology to RNA-binding proteins, specifically to threonyl-tRNA synthetases ${ }^{24}$, and to RNase III family members ${ }^{4}$, respectively. Interestingly, the areas corresponding to RNA binding surfaces in related proteins are oriented towards the solvent and not used for interactions with rRNA. The specific functions of MRPL39 and MRPL44, which is implicated in mitochondrial infantile cardiomyopathy (Extended Data Fig. 9 c) ${ }^{25}$, remain to be established.

A large fraction of mitoribosomes in mammalian mitochondria is tightly bound to the mitochondrial inner membrane independently of the presence of a nascent chain ${ }^{26}$. The position and orientation of MRPL45 next to the ribosomal tunnel exit and the structural features of this protein provide a possible explanation for this observation.

MRPL45 shows homology to the C-terminal domain of TIM44 ${ }^{27}$ and the Saccharomyces cerevisiae mitochondrial protein $\mathrm{Mba} 1^{5}$, both of which are membrane-associated proteins ${ }^{28,29}$. Mba1 functions as a membrane-bound ribosome receptor in yeast ${ }^{8,29}$ and has been crosslinked to ribosomal proteins in the vicinity of the ribosomal exit tunnel ${ }^{9}$. Furthermore, the orientation of MRPL45 on the 39S subunit reveals that the region that is structurally equivalent to the membrane binding segment of TIM44 ${ }^{30}$ is optimally positioned for membrane interactions (Extended Data Fig. 10). Taken together, these findings suggest that mammalian MRPL45 supports the binding of the mitoribosome to the mitochondrial inner membrane to align the 
nascent polypeptide tunnel exit with Oxa1L (Oxa1 in yeast), which functions as an insertase for mitochondrial inner membrane proteins ${ }^{7,31}$.

Although it was previously suggested that a large portion of the 395 subunit is inserted into the membrane ${ }^{11}$, our data on the architecture of the 395 polypeptide exit site indicate that mammalian mitoribosomes are instead anchored to the membrane surface by at least one membrane binding ribosomal protein, obviating the need for the universally conserved SRP membrane targeting machinery ${ }^{7}$.

\section{Proteins functionally replace rRNA}

Comparison of the 39S structure with structures of the prokaryotic ribosome 17,32 shows that the truncation of rRNA segments leads to the formation of deep channels on the surface of the subunit (Fig. $\mathbf{5} \mathbf{a}, \mathbf{b}$ ), some of which are partially occupied by novel mitoribosome-specific protein elements. Although smaller in size compared to the missing bacterial rRNA segments, these proteins probably stabilize the mitoribosomal particle.

A particularly dramatic example of structural remodeling has occurred in the region near MRPL24 (Fig. 5). In the prokaryotic ribosome, ribosomal protein L24 forms extensive contacts to the 23S rRNA helices $\mathrm{H} 7$ and H19 (Fig. 5 b, d) ${ }^{17,32}$. These helices have been lost in the mammalian mitoribosome, eliminating almost all bacterial-like contacts of MRPL24 in the 395 subunit

(Fig. 5 a, c). However, the mitoribosomal-specific protein MRPL45 and two 
currently unassigned additional protein elements form novel contacts with MRPL24, holding it in place with minimal change in its position and orientation compared to the bacterial ribosome (Fig. 5 c). Even though these proteins do not directly replace the missing rRNA structure, they act as an architectural replacement to maintain the position of the functionally important protein MRPL24 ${ }^{7}$ relative to the ribosomal tunnel. Due to the nearly complete remodeling of contacts, this process must have occurred in a stepwise fashion during evolution.

In conclusion, the mitoribosome has undergone a reduction in rRNA content, which is architecturally compensated for by mitoribosome-specific proteins, some of which bear structural and functional homology to soluble and membrane-associated proteins or enzymes. The tunnel exit region is dramatically remodeled, which is probably an adaptation of the 395 subunit to the highly specific requirements of the synthesis and membrane insertion of respiratory chain proteins. Our identification of MRPL45 at the mitoribosomal tunnel exit provides a structural explanation for the observed membrane attachment of mitochondrial ribosomes (Fig. $4 \mathrm{~d}$ ). The results reported here provide an excellent starting point for functional studies.

\section{METHODS SUMMARY}

55 S mitoribosomes and 395 mitoribosomal subunits were prepared from Porcine (Sus scrofa) and bovine (Bos taurus) mitochondria obtained from liver tissue. The three-dimensional structures of the $55 \mathrm{~S}$ mitoribosome and the $39 \mathrm{~S}$ mitoribosomal subunit were determined by cryo-EM. Chemical crosslinking 
followed by mass spectrometry was used to obtain protein-protein crosslinking data. Proteins and rRNA were built into the cryo-EM density based on homology modeling and crosslinking data.

Online Content Any additional Methods, Extended Data display items and Source Data are available in the online version of the paper; references unique to these sections appear only in the online paper.

1 Sagan, L. On the origin of mitosing cells. Journal of Theoretical Biology 14, 255-274 (1967).

2 Desmond, E., Brochier-Armanet, C., Forterre, P. \& Gribaldo, S. On the last common ancestor and early evolution of eukaryotes: reconstructing the history of mitochondrial ribosomes. Res Microbiol 162, 53-70 (2011).

3 Suzuki, T. et al. Structural compensation for the deficit of rRNA with proteins in the mammalian mitochondrial ribosome. Systematic analysis of protein components of the large ribosomal subunit from mammalian mitochondria. J Biol Chem 276, 21724-21736 (2001).

4 Koc, E. C. et al. The large subunit of the mammalian mitochondrial ribosome. Analysis of the complement of ribosomal proteins present. $J$ Biol Chem 276, 43958-43969 (2001).

5 Smits, P., Smeitink, J. A. M., van den Heuvel, L. P., Huynen, M. A. \& Ettema, T. J. G. Reconstructing the evolution of the mitochondrial ribosomal proteome. Nucleic Acids Research 35, 4686-4703 (2007).

6 Attardi, G. \& Ojala, D. Mitochondrial ribosome in HeLa cells. Nature New Biol 229, 133-136 (1971).

7 Ott, M. \& Herrmann, J. M. Co-translational membrane insertion of mitochondrially encoded proteins. Biochim Biophys Acta 1803, 767-775 (2010).

8 Ott, M. et al. Mba1, a membrane-associated ribosome receptor in mitochondria. EMBO J 25, 1603-1610 (2006).

9 Gruschke, S. et al. Proteins at the polypeptide tunnel exit of the yeast mitochondrial ribosome. J Biol Chem 285, 19022-19028 (2010).

$10 \mathrm{Jia}$, L. et al. Yeast Oxa1 interacts with mitochondrial ribosomes: the importance of the C-terminal region of Oxa1. EMBO J 22, 6438-6447 (2003).

11 Agrawal, R. K. \& Sharma, M. R. Structural aspects of mitochondrial translational apparatus. Current Opinion in Structural Biology 22, 797803 (2012).

12 Pearce, S., Nezich, C. L. \& Spinazzola, A. Mitochondrial diseases: translation matters. Mol Cell Neurosci 55, 1-12 (2013). 
13 Sharma, M. R. et al. Structure of the mammalian mitochondrial ribosome reveals an expanded functional role for its component proteins. Cell 115, 97-108 (2003).

14 Mears, J. A. et al. A structural model for the large subunit of the mammalian mitochondrial ribosome. Journal of Molecular Biology 358, 193-212 (2006).

15 Klinge, S., Voigts-Hoffmann, F., Leibundgut, M. \& Ban, N. Atomic structures of the eukaryotic ribosome. Trends Biochem Sci 37, 189-198 (2012).

16 Walzthoeni, T., Leitner, A., Stengel, F. \& Aebersold, R. Mass spectrometry supported determination of protein complex structure. Current Opinion in Structural Biology 23, 252-260 (2013).

17 Polikanov, Y. S., Blaha, G. M. \& Steitz, T. A. How hibernation factors RMF, HPF, and YfiA turn off protein synthesis. Science 336, 915-918 (2012).

18 Smirnov, A., Entelis, N., Martin, R. P. \& Tarassov, I. Biological significance of 5S rRNA import into human mitochondria: role of ribosomal protein MRP-L18. Genes \& Development 25, 1289-1305 (2011).

19 Kelley, L. A. \& Sternberg, M. J. E. Protein structure prediction on the Web: a case study using the Phyre server. Nat Protoc 4, 363-371 (2009).

20 Richter, R. et al. A functional peptidyl-tRNA hydrolase, ICT1, has been recruited into the human mitochondrial ribosome. EMBO J 29, 11161125 (2010).

21 Handa, Y. et al. Solution structure of the catalytic domain of the mitochondrial protein ICT1 that is essential for cell vitality. Journal of Molecular Biology 404, 260-273 (2010).

22 Laurberg, M. et al. Structural basis for translation termination on the $70 S$ ribosome. Nature 454, 852-857 (2008).

23 Gagnon, M. G., Seetharaman, S. V., Bulkley, D. \& Steitz, T. A. Structural basis for the rescue of stalled ribosomes: structure of YaeJ bound to the ribosome. Science 335, 1370-1372 (2012).

24 Spirina, O. et al. Heart-specific splice-variant of a human mitochondrial ribosomal protein (mRNA processing; tissue specific splicing). Gene 261, 229-234 (2000).

25 Carroll, C. J. et al. Whole-exome sequencing identifies a mutation in the mitochondrial ribosome protein MRPL44 to underlie mitochondrial infantile cardiomyopathy. J Med Genet 50, 151-159 (2013).

26 Liu, M. \& Spremulli, L. Interaction of mammalian mitochondrial ribosomes with the inner membrane. J Biol Chem 275, 29400-29406 (2000).

27 Schneider, H. C. et al. Mitochondrial Hsp70/MIM44 complex facilitates protein import. Nature 371, 768-774 (1994).

28 Weiss, C. et al. Domain structure and lipid interaction of recombinant yeast Tim44. Proc Natl Acad Sci USA 96, 8890-8894 (1999).

29 Preuss, M. et al. Mba1, a novel component of the mitochondrial protein export machinery of the yeast Saccharomyces cerevisiae. The Journal of Cell Biology 153, 1085-1096 (2001). 
30 Cui, W., Josyula, R., Li, J., Fu, Z. \& Sha, B. Membrane binding mechanism of yeast mitochondrial peripheral membrane protein TIM44. Protein Pept Lett 18, 718-725 (2011).

31 Stiburek, L. et al. Knockdown of human Oxa1l impairs the biogenesis of F1Fo-ATP synthase and NADH:ubiquinone oxidoreductase. Journal of Molecular Biology 374, 506-516 (2007).

32 Ban, N., Nissen, P., Hansen, J., Moore, P. B. \& Steitz, T. A. The complete atomic structure of the large ribosomal subunit at $2.4 \mathrm{~A}$ resolution. Science 289, 905-920 (2000).

Supplementary Information is linked to the online version of the paper at www.nature.com/nature.

Acknowledgments Cryo-EM data was collected at the electron microscopy facility of ETH Zurich (EMEZ) and at FEI Company Eindhoven. We thank Peter Tittmann (EMEZ) and Felix de Haas and Kasim Sader (FEI Company) for support. Acknowledge Computing Resources. This work was supported by the Swiss National Science Foundation (SNSF), the National Center of Excellence in Research (NCCR) Structural Biology program of the Swiss National Science Foundation (SNSF), European Research Council (ERC) grant 250071 under the European Community's Seventh Framework Programme (to N.B.), the Commission of the European Communities through the PROSPECTS consortium (EU FP7 projects 201648, 233226) (R.A.), and the European Research Council (ERC-2008-AdG 233226) (R.A.).

Author Contributions F.V.-H., J.P.E. and N.B. initiated the project; F.V.-H. and J.P.E. established the purification procedures. F.V.-H., B.G. and P.B. performed preparation of the mitoribosomes. B.G., P.B., and D.B. prepared cryo-EM samples. D.B. acquired the cryo-EM data. B.G., D.B., and P.B. calculated the cryo-EM reconstructions. M.L., B.G., P.B., D.B., and N.B. 
interpreted the structure. A.L. performed CX-MS experiments in the laboratory of R.A.. All authors contributed to the final version of the paper.

Author Information The cryo-EM map of the 39S mitoribosomal subunit has been deposited in the Electron Microscopy Databank with accession code EMD-2490. The coordinates of the cryo-EM based model of the 39S mitoribosomal subunit have been deposited in the protein databank with PDB code 4CE4. Reprints and permissions information is available at www.nature.com/reprints. The authors declare that they have no competing financial interests. Correspondence and requests for materials should be addressed to N.B. (ban@mol.biol.ethz.ch) or R.A. (aebersold@imsb.biol.ethz.ch). 


\section{METHODS}

Preparation of porcine and bovine mitochondria. Porcine and bovine mitochondria were prepared from livers of freshly slaughtered pigs (Sus scrofa) and cows (Bos taurus). Livers were transported immersed in liver buffer (10 mM HEPES-KOH pH 7.6, 340 mM sucrose, 1 mM EDTA) on ice. 6 $\mathrm{kg}$ of livers (5 livers) were cut into small pieces after removal of the gall bladder and connective tissue. The pieces were washed in isotonic buffer (10 mM HEPES-KOH pH 7.6, 70 mM sucrose, 220 mM mannitol, 1 mM EDTA; 2 $\mathrm{L}$ of isotonic buffer per $1 \mathrm{~kg}$ of liver tissue). The liver was then ground into isotonic buffer using a meat grinder and diluted to a total volume of $12 \mathrm{~L}$ using isotonic buffer. The suspension was homogenized using an Ultra-Turrax (IKA, Staufen, Germany) homogenizer (40 sec), filtered through one layer of gauze, and diluted to a total volume of $18 \mathrm{~L}$ with isotonic buffer. The resulting suspension was centrifuged ( $600 \mathrm{rcf}, 15 \mathrm{~min}, 4^{\circ} \mathrm{C}$ ) using a Sorvall SLC-6000 rotor (Thermo Fisher Scientific, Waltham, MA, USA). The supernatant was decanted and again centrifuged using a SLC-6000 rotor (5'900 rcf, 24 min, $\left.4^{\circ} \mathrm{C}\right)$. The pellets were resuspended in a total of $8 \mathrm{~L}$ of isotonic buffer and again filtered through one layer of gauze and homogenized using an UltraTurrax homogenizer (30 sec). After dilution to $12 \mathrm{~L}$, the suspension was centrifuged (SLC-6000, 5'600 rcf, $24 \mathrm{~min}, 4^{\circ} \mathrm{C}$ ), and the pellets were again resuspended in isotonic buffer ( $4 \mathrm{~L}$ total volume).

The suspension was treated with digitonin $(0.1 \mathrm{~g} / \mathrm{L})$ for $15 \mathrm{~min}$ and diluted to $12 \mathrm{~L}$ using isotonic buffer. The resulting mitoplasts were concentrated by centrifugations in decreasing amounts of isotonic buffer $(2 \times$ SLC-6000, 
5'900 rcf, $4^{\circ} \mathrm{C} ; 2$ x Sorvall SLA-1500 (Thermo Fisher Scientific, Waltham, MA, USA), $13^{\prime} 000 \mathrm{rpm}, 20 \mathrm{~min}, 4^{\circ} \mathrm{C}$ ) and the pellet of the final step was flashfrozen in liquid nitrogen and stored at $-80^{\circ} \mathrm{C}$.

Preparation of $55 \mathrm{~S}$ mitoribosomes. $100 \mathrm{~g}$ of frozen mitoplasts were thawed in $150 \mathrm{~mL}$ lysis buffer (20 mM HEPES-KOH pH 7.6, $100 \mathrm{mM} \mathrm{KCl,} 20 \mathrm{mM}$ $\mathrm{MgCl}_{2}, 1 \mathrm{mM}$ DTT, $125 \mu \mathrm{M}$ spermine, $125 \mu \mathrm{M}$ spermidine) and brought to a total volume of $337.5 \mathrm{~mL}$ with monosome buffer (20 mM HEPES-KOH pH 7.6, $100 \mathrm{mM} \mathrm{KCl}, 1 \mathrm{mM}$ DTT, $20 \mathrm{mM} \mathrm{MgCl}$ ). $37.5 \mathrm{~mL}$ Triton X-100 buffer (20 mM HEPES-KOH pH 7.6, $100 \mathrm{mM} \mathrm{KCl,} 1 \mathrm{mM}$ DTT, 20 mM MgCl $2,16 \%$ (v/v) Triton $\mathrm{X}-100$ ) were added and the solution was stirred for 15 min at $4^{\circ} \mathrm{C}$ before homogenization using a Dounce homogenizer. The suspension was centrifuged (SLA-1500, 13'000 rpm, $20 \mathrm{~min}, 4^{\circ} \mathrm{C}$ ), and the supernatant was PEG precipitated in 5\% (w/v) PEG 10 '000 for $15 \mathrm{~min}$. The precipitate was collected by centrifugation (Sorvall SLA-3000 (Thermo Fisher Scientific, Waltham, MA, USA), $2500 \mathrm{rcf}, 7 \mathrm{~min}, 4^{\circ} \mathrm{C}$ ). Each pellet was resuspended in $35 \mathrm{~mL}$ monosome buffer ( $2 \mathrm{~h}$ shaking), and the suspension was homogenized using a Dounce homogenizer before centrifugation using a Beckman (Beckman-Coulter Intl., Nyon, Switzerland) Type 45Ti rotor (28'000 rpm, 17 $\left.\min , 4^{\circ} \mathrm{C}\right)$. The supernatant was loaded onto $50 \%(\mathrm{w} / \mathrm{v})$ sucrose cushions $(15$ $\mathrm{mL}$ ) and centrifuged (Beckman Type 70Ti (Beckman-Coulter Intl., Nyon, Switzerland), $50^{\prime} 000 \mathrm{rpm}, 24 \mathrm{~h}, 4^{\circ} \mathrm{C}$ ). Pellets were dissolved in $500 \mu \mathrm{L}$ monosome buffer (shaking $230 \mathrm{rpm}, 1 \mathrm{~h}$ ) and cleared (tabletop centrifuge, $\left.16{ }^{\prime} 000 \mathrm{rpm}, 20 \mathrm{~min}, 4^{\circ} \mathrm{C}\right)$. The sample was distributed onto $10 \%-40 \%(\mathrm{w} / \mathrm{v})$ sucrose gradients ( $1 \mathrm{~mL}$ per gradient) and centrifuged (Beckmann SW-32 Ti 
(Beckman-Coulter Intl., Nyon, Switzerland), 26’000 rpm, 12 h, 4 C). The gradients were fractionated and fractions corresponding to the $55 \mathrm{~S}$ mitoribosome were collected, and the buffer was exchanged to sucrose-free monosome buffer using Amicon Ultra-4 centrifugal filter units (100’000 MWCO) (EMD Millipore Corp., Billerica, MA, USA). The sample was concentrated and subjected to gel filtration on a HiLoad Superdex 75 prep grade size exclusion column (GE Healthcare, Freiburg, Germany). The 55S peak was pooled, concentrated, and flash-frozen in liquid nitrogen.

Preparation of 39S mitoribosomal subunits. Preparation of 395 mitoribosomal subunits was performed as described for $55 \mathrm{~S}$ mitoribosomes, with minor modifications: The pellets of the sucrose cushion ultracentrifugation were resuspended in dissociation buffer (20 mM HEPES$\mathrm{KOH} \mathrm{pH} 7.6,300 \mathrm{mM} \mathrm{KCl}, 1 \mathrm{mM}$ DTT, $5 \mathrm{mM} \mathrm{MgCl}_{2}$ ), and the sucrose gradients were prepared in dissociation buffer.

\section{Cryo-EM sample preparation and data acquisition. Samples were} prepared on Quantifoil holey carbon copper grids (R 2/1). $5 \mu \mathrm{L}$ of sample containing $40 \mathrm{nM}$ of $39 \mathrm{~S}$ mitoribosomal subunit in monosome buffer $(\mathrm{pH} 7.9)$ were applied onto the grid, and the grid was flash-frozen by plunging into liquid ethane after manual blotting. Cryo-EM dataset I (1'034'220 particle images) was collected on a FEI Titan Krios cryo-transmission electron microscope (TEM) equipped with a FEI Falcon I direct electron detection device (FEI Company, Hillsboro, OR, USA) at 99'290 x magnification, resulting in a pixel size of $1.41 \AA$ on the object scale. Data was acquired in a 
fully automated manner in spot scans of 3 or 4 spots per ice hole using the FEI EPU software (FEI Company, Hillsboro, OR, USA). Cryo-EM dataset II (626'664 particle images) was collected on a FEI Titan Krios cryo-TEM equipped with a backthinned FEI Falcon II direct electron detection device (FEI Company, Hillsboro, OR, USA) at 141'510 x magnification, resulting in a pixel size of $1.06 \AA$ on the object scale. Data was acquired automatically in spot scans of 5 spots per ice hole using the FEI EPU software.

Image processing. Particle images were selected and extracted using batchboxer (EMAN 1.6) ${ }^{33}$. Defocus determination and CTF correction ${ }^{34}$ were performed using SPIDER ${ }^{35}$. The particle images collected using the FEI Falcon II camera were scaled to match the lower magnification of the data collected using the FEI Falcon I. The initial reference for the reconstruction of the 395 mitoribosomal subunit was extracted from a cryo-EM reconstruction of the 555 mitoribosome, which in turn was obtained from a small dataset of $55 S$ mitoribosomal particles processed in IMAGIC $5^{36}$, using an E. coli $70 S$ ribosome (EMDB accession code EMD-1615) as initial reference. The dataset was subjected to computational sorting by multiparticle refinement with up to four reference volumes ${ }^{37}$ using an initial seed of two reference volumes corresponding to two particle subpopulations. The seed volumes were computed based on bootstrap 3D variance classification. This procedure yielded a data subset of $627^{\prime} 730$ particle images, corresponding to class 1 of the multiparticle classification (Extended Data Fig. 1 b). 
The reconstruction of the 395 mitoribosomal subunit was refined to higher resolution by projection matching in SPIDER ${ }^{35}$ employing a gold standard FSC refinement procedure ${ }^{38}$. To this end, the initial 395 reference was lowpass filtered to approximately $20 \AA$ resolution, and particle half sets of 313'865 particle images each were aligned onto this initial reference, resulting in two reconstructions. These two reconstructions were refined to higher resolution fully independently using only the corresponding data half set. During later stages of refinement, the Fourier amplitudes of the input volumes were enhanced based on the small angle X-ray scattering amplitude curve of ribosomes ${ }^{39}$. Throughout the refinement, excess particle images from overrepresented views were excluded from the calculation of 3D volumes to avoid distortion of the reconstructions. Therefore, only about 232'181 of the total $627^{\prime} 730$ particle images contributed to the final 3D map. The final rounds of refinement were performed using particle images at the full resolution of the Falcon I data (1.41 $\AA$ per pixel at the object scale) with both reference volumes low-pass filtered to approximately $6 \AA$ resolution. The resolution was determined using the $\mathrm{FSC}=0.143$ criterion ${ }^{38}$, resulting in a resolution estimate of $4.9 \AA$ (Extended Data Fig. 1 a). The use of the $\mathrm{FSC}=0.143$ criterion is justified because the gold standard refinement procedure eliminates accumulation of high-resolution noise by alignment bias during the refinement process ${ }^{38}$. For visualization purposes, the final cryo-EM map was amplitude- and MTF-corrected using RELION ${ }^{38}$.

Chemical crosslinking of S. scrofa 39S subunits and B. taurus 55 S

ribosomes. The ribosome preparation $(200 \mu \mathrm{L}$ at an approximate 
concentration of $0.35 \mathrm{mg} / \mathrm{mL}$ ) was cross-linked in $20 \mathrm{mM} \mathrm{HEPES-KOH} \mathrm{(pH}$ 7.6) containing $100 \mathrm{mM} \mathrm{KCl}, 20 \mathrm{mM} \mathrm{MgCl}_{2}, 1 \mathrm{mM}$ DTT, $10 \%$ (w/v) sucrose by adding disuccinimidyl suberate- $\mathrm{d}_{0} / \mathrm{d}_{12}(25 \mathrm{mM}$ stock solution in anhydrous dimethyl formamide; Creative Molecules Inc., Victoria, BC, Canada) to a final concentration of $0.2 \mathrm{mM}$. The crosslinking reaction was allowed to proceed for 30 min at $37^{\circ} \mathrm{C}$ before quenching by addition of $1 \mathrm{M}$ ammonium bicarbonate stock solution to a final concentration of $50 \mathrm{mM} \mathrm{NH}_{4} \mathrm{HCO}_{3}$. Crosslinked ribosome proteins were reduced, alkylated and enzymatically digested as described previously ${ }^{40}$ with the exception that a two-step digestion procedure with endoproteinase Lys-C (Wako Chemicals, 3 h, enzyme-to-substrate ratio 1:100) and trypsin (Promega, overnight, enzyme-to-substrate ratio 1:50) was used. The digested mixture was fractionated by size exclusion chromatography (SEC) as desribed previously ${ }^{40}$. Two fractions corresponding to elution volumes of 1.0-1.1 mL and 1.1-1.2 mL were collected and evaporated to dryness in a vacuum centrifuge.

\section{Liquid chromatography-tandem mass spectrometry (LC-MS/MS)} analysis of crosslinked ribosomal subunits. The SEC fractions were resuspended in water/acetonitrile/formic acid (95:5:0.1, v/v/v) and aliquots corresponding to an estimated $1 \mu \mathrm{g}$ were used for LC-MS/MS using an EasynLC 1000 LC system connected to an Orbitrap Elite mass spectrometer equipped with a Nanoflex electrospray ion source (all Thermo Scientific). Both SEC fractions were analyzed in duplicate.

Peptides were separated on an Acclaim PepMap RSLC column (15 cm x 75 $\mu \mathrm{m}, 2 \mu \mathrm{m}$ particle size, Thermo Scientific) at a flow rate of $300 \mathrm{~nL} / \mathrm{min}$ using 
the following gradient: 0-90 $\min , 9-35 \% \mathrm{~B} ; 90-92 \mathrm{~min}, 35-80 \% \mathrm{~B} ; 92-102 \mathrm{~min}$, $80 \% \mathrm{~B}$; where solvent $A=$ water/acetonitrile/formic acid $(95: 5: 0.1, \mathrm{v} / \mathrm{v} / \mathrm{v})$ and $B$ $=$ acetonitrile/water/formic acid (95:5:0.1, v/v/v).

The mass spectrometer was programmed to acquire MS/MS spectra in datadependent mode, selecting the ten most abundant precursor ions for fragmentation per cycle and the following settings: MS detection in the orbitrap, scan range $350-1600 \mathrm{~m} / \mathrm{z}$, resolution 120'000; MS/MS detection in the linear ion trap, isolation width $2.0 \mathrm{~m} / \mathrm{z}$, normalized collision energy $35 \%$. Precursors of charge states +1 and +2 as well as precursors of unknown charge states were excluded from fragmentation. Dynamic exclusion was activated with exclusion duration of $30 \mathrm{sec}$, a repeat count of 1 and a list size of 500 .

Data analysis of LC-MS/MS data. Mass spectrometry data acquired in Xcalibur .raw format (Thermo Scientific) were converted into mzXML format using ProteoWizard/msconvert ${ }^{41}$ and searched against a database of the $S$. scrofa 395 mitoribosomal subunit protein sequences using an in-house installation of $x Q_{\text {Quest }}{ }^{42}$. The sequence database was compiled from S. scrofa mitoribosomal protein sequences identified by protein-protein BLAST (on http://blast.ncbi.nlm.nih.gov/) ${ }^{43}$ using the annotated human protein sequences obtained from the Ribosomal Protein Gene database ${ }^{44}$ as input for the BLAST searches. If no homologs could be identified using protein BLAST, an additional BLAST search of the human MRP sequences against a translated nucleotide database (nucleotide collection on 
http://blast.ncbi.nlm.nih.gov/; limited to S. scrofa sequences) was conducted. The $B$. taurus protein data set was compiled from proteins assigned to the bovine mitochondrial ribosome in Uniprot ${ }^{45}$. $x$ Quest search parameters were as follows: enzyme $=$ trypsin, maximum number of missed cleavages $=2$, mass shift of cross-linker addition $=138.068080 \mathrm{Da}$, initial mass tolerance $=$ $15 \mathrm{ppm}$. Results were further filtered according to the following criteria: minimum peptide length $=6$ amino acids, mass tolerance $=5 \mathrm{ppm}$, fraction of total ion current assigned $(\% \mathrm{TIC})>0.1$, delta score $<0.9$, minimum number of cleavages per peptide $=4$. False discovery rates were determined using xProphet ${ }^{46}$, and determined to be less that $3 \%$ for the selected score threshold of 25 .

Structural modeling. The rRNA model was built using $\mathrm{COOT}^{47}$ and $\mathrm{O}^{48}$. The structure of the $T$. thermophilus $23 \mathrm{~S}$ rRNA ${ }^{17}$ served as a reference and was docked into the cryo-EM map. Structural elements present the cryo-EM map were retained, while structural elements absent from the cryo-EM density were removed from the model. Bases were mutated in $\mathrm{COOT}^{47}$ to the $S$. scrofa sequence (GenBank accession code AJ002189) according to multiple and pairwise sequence alignments ${ }^{49}$ and the secondary structure predictions for the human ${ }^{49}$ and bovine ${ }^{14} 16 S$ rRNA. Rigid body fitting of segments of the model were performed in $\mathrm{O}^{48}$. Extensions and further adjustments to the model as well as regularization were performed in $\mathrm{COOT}^{47}$. Our final model of the 16S rRNA (model chain A) contains 1443 of the total 1569 nucleotides of the S. scrofa 16 S rRNA sequence (92\% coverage; $98 \%$ coverage if the nucleotides of the $L 1$ and $L 7 / L 12$ stalks that are not built due to flexibility are 
excluded from the total). The sequence for residues $24-27$ and $35-37$ in domain I remains unassigned. Our model of the RNA of the 395 central protuberance (model chain B) contains 29 nucleotides, but without assigned sequence. Where the sequence could not be assigned, the structure has been deposited as poly-phosphates. Since purines could not be distinguished from pyrimidines in the $4.9 \AA$ cryo-EM map, we cannot fully exclude register shifts in poorly conserved or peripheral regions of the structure.

Because de-novo tracing of protein folds is not possible at the resolution of our cryo-EM map, homology models of the mitoribosomal proteins were obtained using the Phyre2 web server ${ }^{19}$. For proteins with homologs in the bacterial 50 S subunit, the $T$. thermophilus structure ${ }^{17}$ served as template for modeling in pairwise mode, while homologs for several other mitoribosomal proteins were detected and used as models by searching the entire PDB database (Supplementary Table 1). The resulting models were either docked into the cryo-EM density according to their position in the bacterial $50 \mathrm{~S}$ subunit, or fitted based on the features of the cryo-EM density. In this case, their position was cross-checked using CX-MS data if no bacterial homologs exist. Adjustments of the homology models to the density were performed in $\mathrm{O}^{48}$ if required. For a few docked proteins, extensions of the core fold were clearly visible in the EM density and were built as unassigned "UNK" residues using the secondary structure predictions from Phyre ${ }^{19}$ as a guide. Several areas of EM-density clearly showed the features of isolated $\alpha$-helices, into which standard UNK helices could be docked. One entire thioredoxin fold was clearly recognizable in the EM density. However, because two of the currently 
known mitoribosomal proteins adopt this fold and due to the lack of crosslinking data for these proteins, an unassigned UNK model of the homologous NMR structure (PDB ID 1S3A) was fitted into the density. Both the UNK helices and the thioredoxin fold are indicated in all figures as dark grey models. The final model containing both the rRNAs and the proteins was regularized using six cycles of energy minimization in PHENIX ${ }^{50}$ while applying Ramachandran and secondary structure restraints.

Figures. Figures of molecular structures were created using UCSF Chimera ${ }^{51}$. Figure 3 representing the CX-MS based protein crosslink network was generated using Cytoscape ${ }^{52}$.

33 Ludtke, S. J., Baldwin, P. R. \& Chiu, W. EMAN: semiautomated software for high-resolution single-particle reconstructions. Journal of Structural Biology 128, 82-97 (1999).

34 Mindell, J. A. \& Grigorieff, N. Accurate determination of local defocus and specimen tilt in electron microscopy. Journal of Structural Biology 142, 334-347 (2003).

35 Frank, J. et al. SPIDER and WEB: processing and visualization of images in 3D electron microscopy and related fields. Journal of Structural Biology 116, 190-199 (1996).

36 Van Heel, M., Harauz, G., Orlova, E. V., Schmidt, R. \& Schatz, M. A new generation of the IMAGIC image processing system. Journal of Structural Biology 116, 17-24 (1996).

37 Loerke, J., Giesebrecht, J. \& Spahn, C. M. T. Multiparticle cryo-EM of ribosomes. Meth Enzymol 483, 161-177 (2010).

38 Scheres, S. H. W. RELION: Implementation of a Bayesian approach to cryo-EM structure determination. Journal of Structural Biology 180, 519-530 (2012).

39 Gabashvili, I. S. et al. Solution structure of the E. coli 70 S ribosome at 11.5 A resolution. Cell 100, 537-549 (2000).

40 Leitner, A. et al. Expanding the chemical cross-linking toolbox by the use of multiple proteases and enrichment by size exclusion chromatography. Mol Cell Proteomics 11, M111.014126 (2012).

41 Chambers, M. C. et al. A cross-platform toolkit for mass spectrometry and proteomics. Nat Biotechnol 30, 918-920 (2012).

42 Rinner, O. et al. Identification of cross-linked peptides from large sequence databases. Nat Methods 5, 315-318 (2008). 
43 Altschul, S. F., Gish, W., Miller, W., Myers, E. W. \& Lipman, D. J. Basic local alignment search tool. J Mol Biol 215, 403-410 (1990).

44 Nakao, A., Yoshihama, M. \& Kenmochi, N. RPG: The Ribosomal Protein Gene Database. Nucleic Acids Res. 32, 168-170 (2004).

45 UniProtConsortium. Ongoing and future developments at the Universal Protein Resource. Nucleic Acids Research 39, D214-219 (2011).

46 Walzthoeni, T. et al. False discovery rate estimation for cross-linked peptides identified by mass spectrometry. Nat Meth 9, 901-903 (2012).

47 Emsley, P., Lohkamp, B., Scott, W. G. \& Cowtan, K. Features and development of Coot. Acta Crystallogr D Biol Crystallogr 66, 486-501 (2010).

48 Jones, T. A., Zou, J. Y., Cowan, S. W. \& Kjeldgaard, M. Improved methods for building protein models in electron density maps and the location of errors in these models. Acta Crystallogr, A, Found Crystallogr 47 (Pt 2), 110-119 (1991).

49 Cannone, J. J. et al. The comparative RNA web (CRW) site: an online database of comparative sequence and structure information for ribosomal, intron, and other RNAs. BMC Bioinformatics 3, 2 (2002).

50 Adams, P. D. et al. PHENIX: a comprehensive Python-based system for macromolecular structure solution. Acta Cryst D 66, 213-221 (2012).

51 Pettersen, E. F. et al. UCSF Chimera--a visualization system for exploratory research and analysis. J Comput Chem 25, 1605-1612 (2004).

52 Cline, M. S. et al. Integration of biological networks and gene expression data using Cytoscape. Nat Protoc 2, 2366-2382 (2007).

53 Yusupov, M. M. et al. Crystal structure of the ribosome at $5.5 \mathrm{~A}$ resolution. Science 292, 883-896 (2001).

54 Josyula, R., Jin, Z., Fu, Z. \& Sha, B. Crystal structure of yeast mitochondrial peripheral membrane protein Tim44p C-terminal domain. Journal of Molecular Biology 359, 798-804 (2006). 

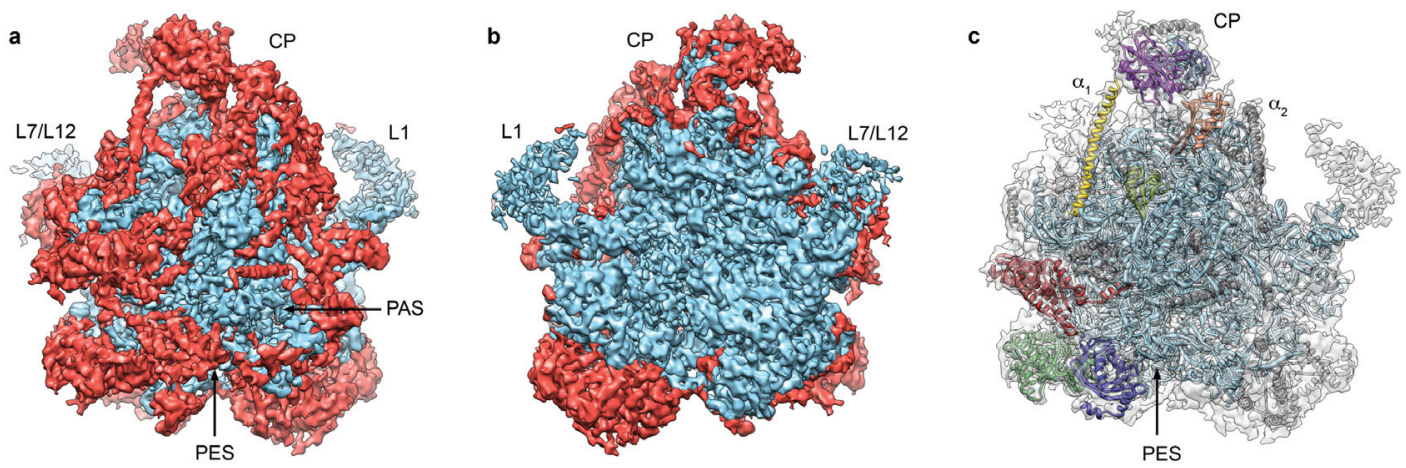

Figure 1 | Cryo-EM reconstruction of the mitoribosomal 39S subunit. a, Solvent side and

b, subunit interface side of the 395 subunit segmented into conserved (blue) and mitoribosome-specific (red) density elements. CP, central protuberance; L7/L12, L7/L12 stalk base; L1, L1 stalk; PES, polypeptide exit site; PAS, polypeptide accessible site. c, Two clusters of mitochondrial-specific ribosomal proteins (colored) at the central protuberance (CP) and the polypeptide exit site (PES). The CP is flanked by prominent $\alpha$-helices ( $\alpha_{1}$ and

$a_{2}$ ). Conserved parts of the 39S subunit - light blue; unassigned protein segments - dark grey. 
a

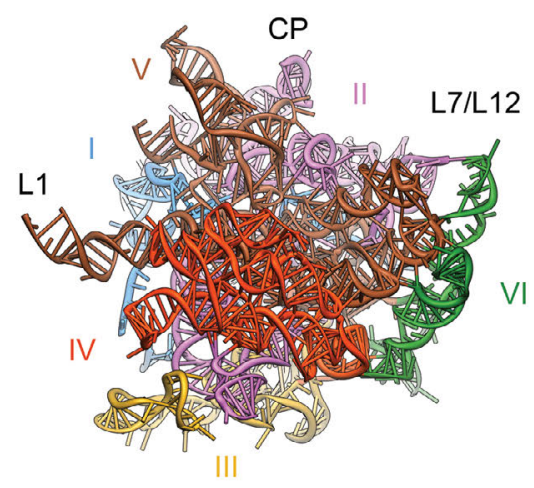

b

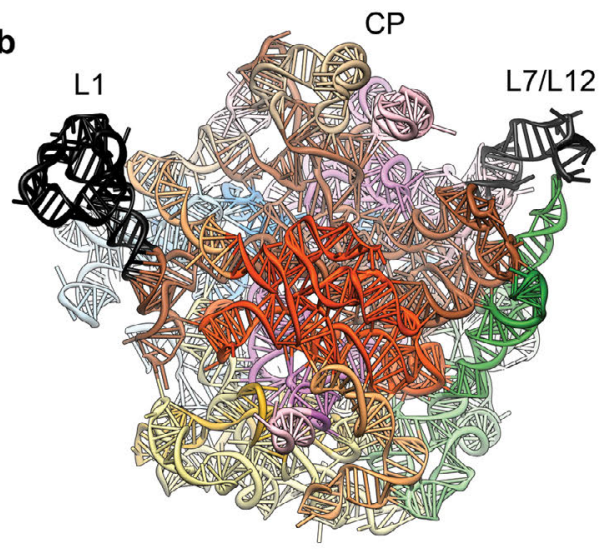

C

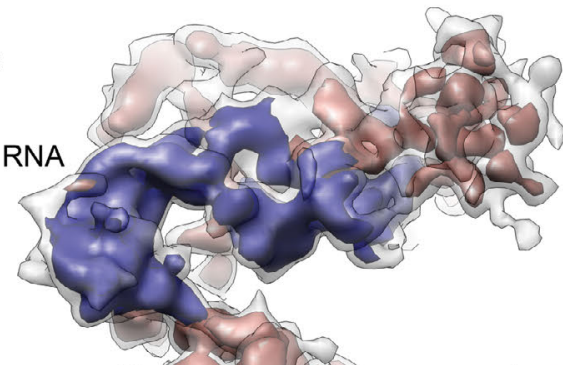

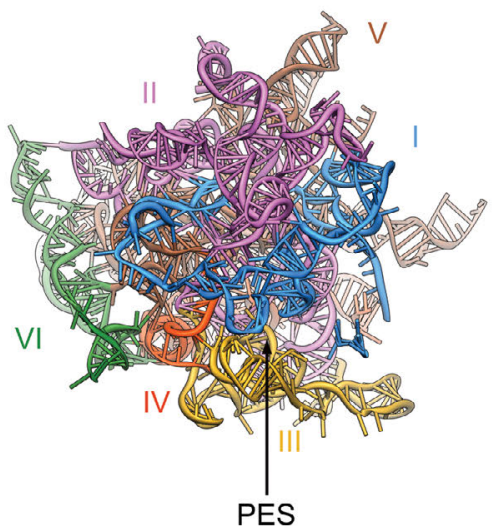

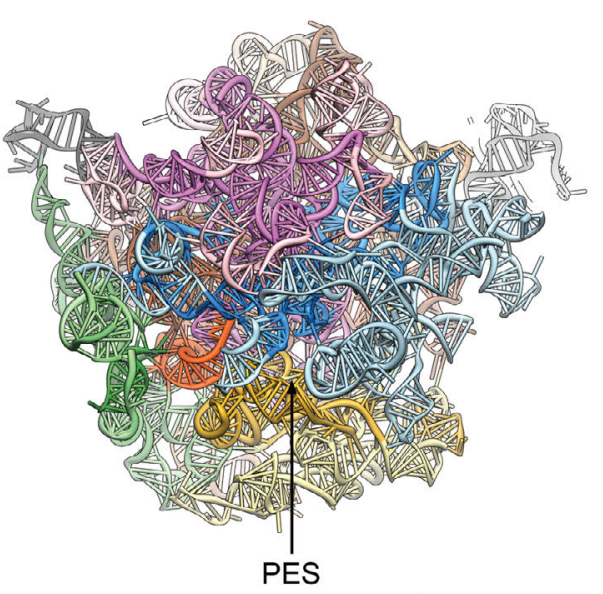

PES

d

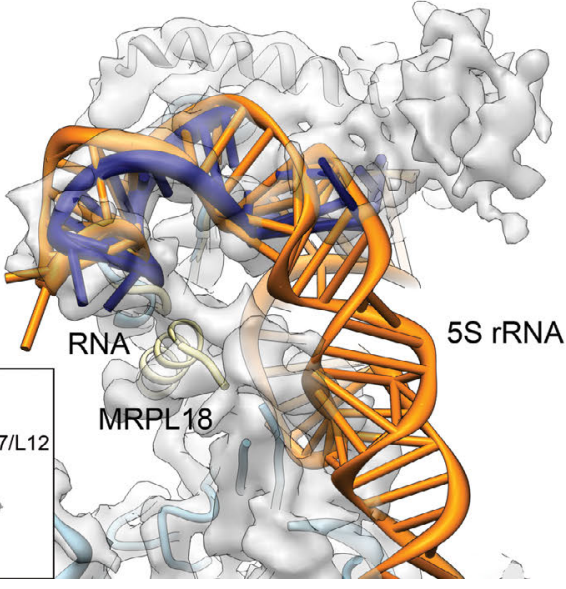

Figure 2 | The ribosomal RNA of the 395 mitoribosomal subunit. a, Domain architecture of the mitoribosomal 16S rRNA (left, subunit interface side; right, solvent side). Domains I-VI are color coded, as indicated by Roman numerals. b, Bacterial 23S rRNA structure (PDB ID 3V2D) ${ }^{17}$. Color code as in $\mathbf{a}$, with segments absent in the mammalian mitoribosome shown in lighter colors (black: segments not modeled in the 39S subunit due to disorder). c, A clear rRNA major groove feature visualized in the cryo-EM map indicates the presence of a second 
rRNA molecule (blue) in the 39S subunit. d, Comparison of this mitochondrial rRNA (blue) and the bacterial 5S rRNA (orange; superposition according to MRPL18/L18). 


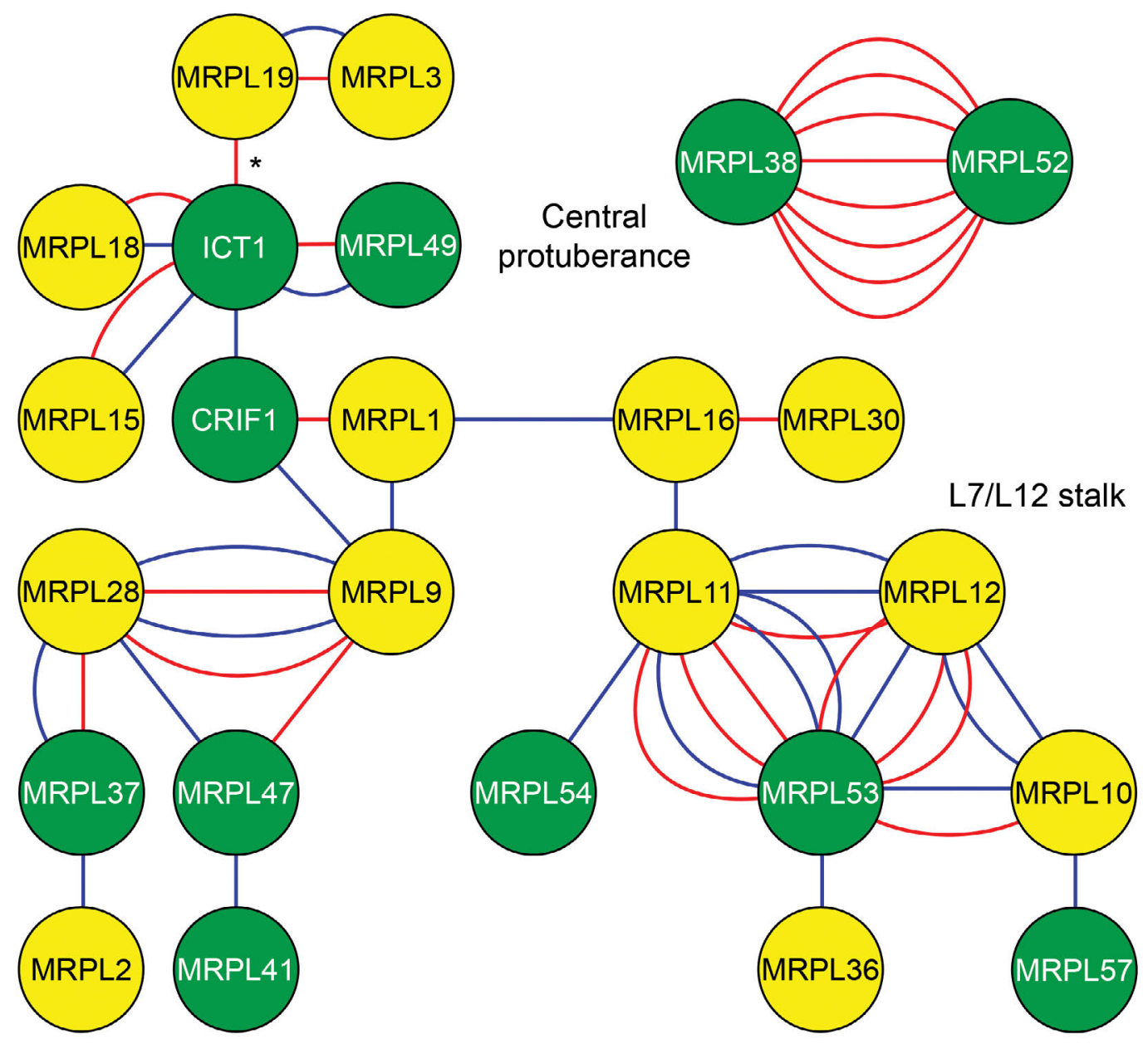

Polypeptide tunnel exit

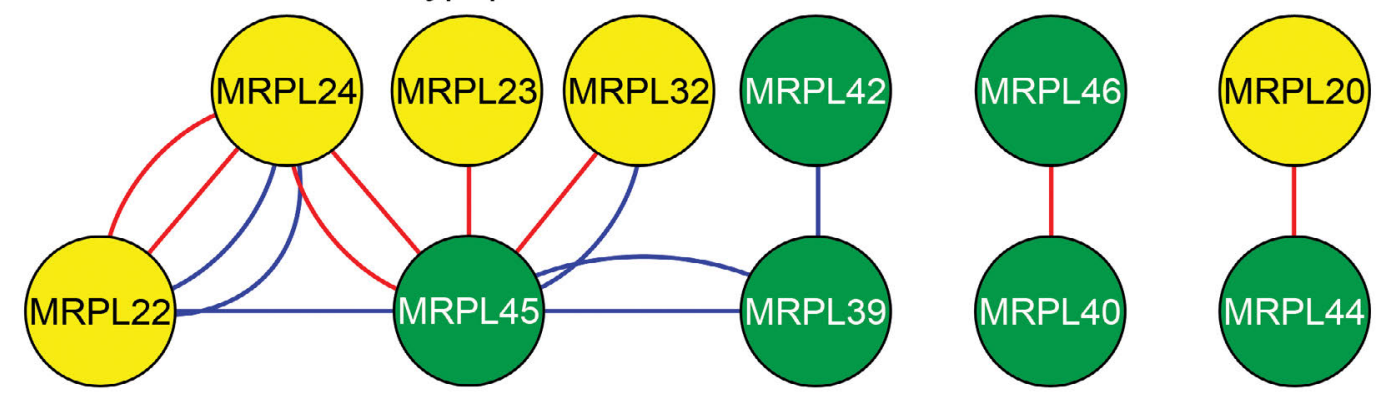

Figure 3 | Overview of the inter-protein crosslinks of 39S mitoribosomal subunit

proteins. Statistically significant inter-protein crosslink hits of 395 subunit proteins (xQuest Id score > 25) obtained by CX-MS experiments. Mitoribosomal proteins with bacterial ribosomal homologs are shown as yellow nodes, those without homologs in bacterial ribosomes in green. Crosslinks obtained using S. scrofa 39S subunits are shown as red lines, crosslinks obtained using Bos taurus 55S mitoribosomes are shown in blue. The crosslink of ICT1 with MRPL19 (asterisk) in S. scrofa is likely a false positive identification (see Supplementary Table 3). 


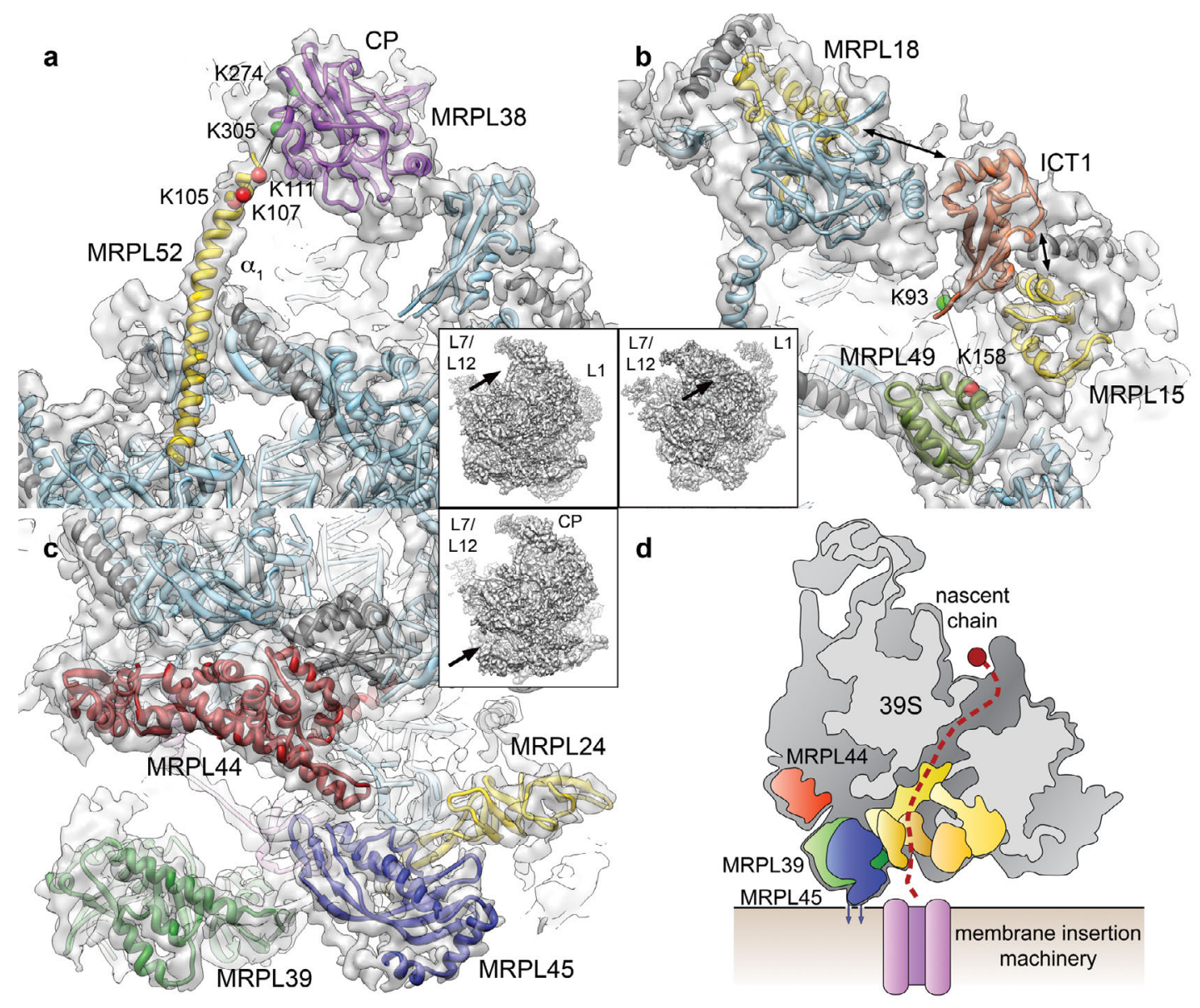

Figure 4 | Structures of novel mitochondrial-specific proteins at the highly remodeled mitoribosomal central protuberance and at the polypeptide tunnel exit. a, Crosslinks between MRPL38 and MRPL52 allow assignment of MRPL52 to helical density $\alpha_{1}$ (crosslinked residues shown as spheres if modeled, or as double arrows otherwise). Insets show the whole 395 subunit for orientation. b, ICT1 (orange) and MRPL49 (olive) crosslink to each other and can be fitted into the cryo-EM map at the CP. ICT1 also crosslinks to MRPL15 and MRPL18 (gold). c, Tunnel exit region of the 39S subunit with fitted MRPL39 (green), MRPL44 (red), and MRPL45 (blue). d, Schematic illustration of the 395 subunit bound to the mitochondrial inner membrane. 

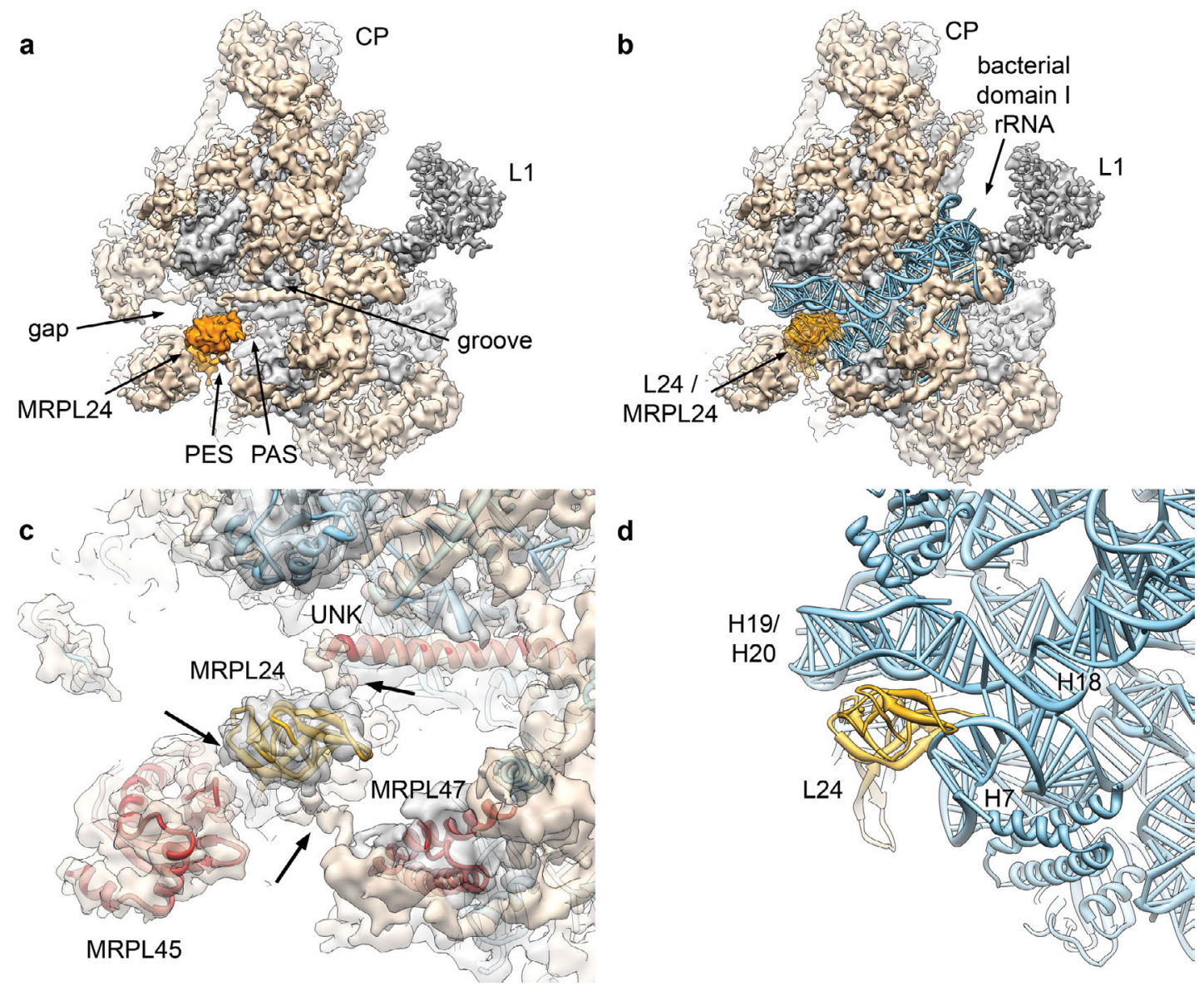

Figure 5 | Remodeling of MRPL24 contact sites in the 395 subunit. a, A deep groove runs above MRPL24, caused by extensive loss of rRNA compared to bacterial $23 S$ rRNA (conserved parts grey; mitochondrial-specific parts brown; MRPL24 gold). b, 39S cryo-EM map shown together with a part of domain I of the bacterial $23 \mathrm{~S}$ rRNA ${ }^{17}$ (PDB ID 3V2D) (blue). c, Novel contacts (arrows) of mitoribosomal protein elements (red), among them MRPL45, additional density near MRPL47, and an $\alpha$-helical density emanating from the core of the subunit (UNK), stabilize MRPL24. d, These mitochondrial specific contacts of MRPL24 replace the extensive interactions of L24 with 23S rRNA in bacteria (colors as in b; labeled rRNA helices are missing in the 395 subunit). 
a

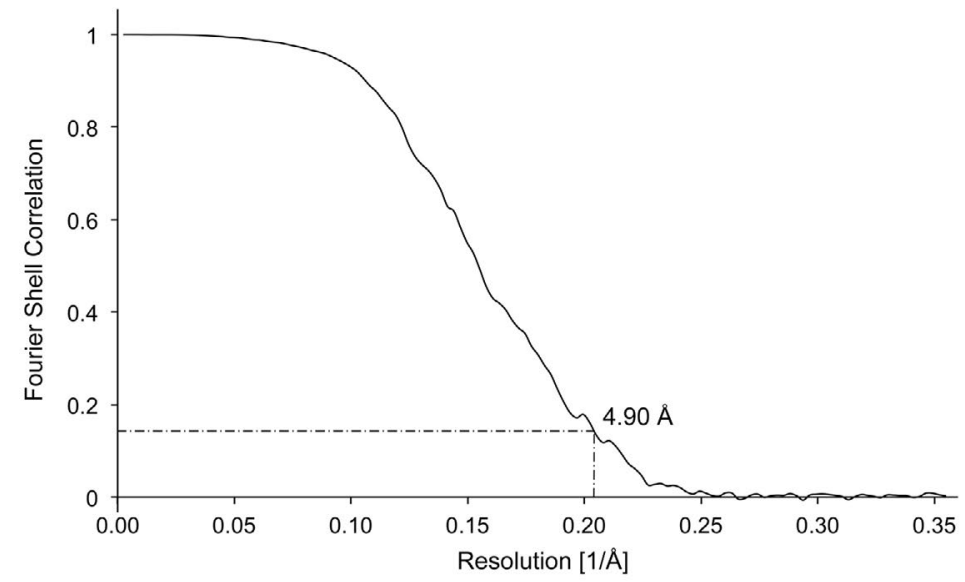

b

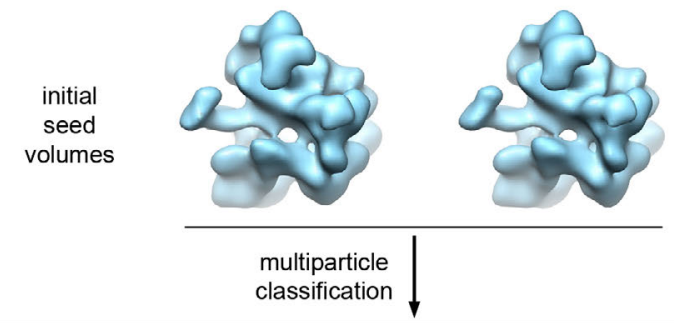

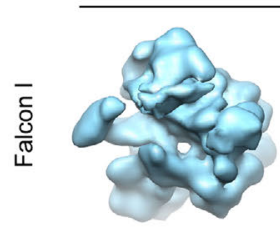

376329

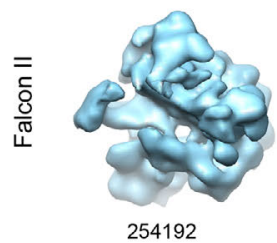

class 1

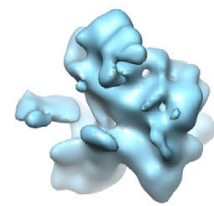

147763

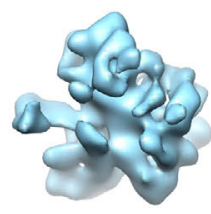

108689

class 2

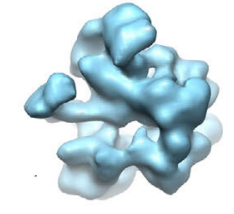

175057

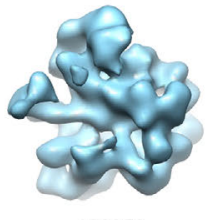

106050

class 3
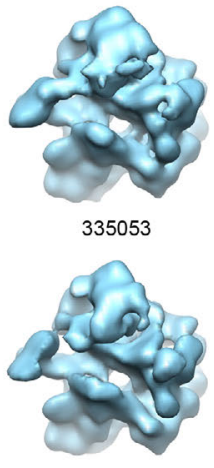

151755

class 4

Extended Data Figure 1 | Refinement of the 39S mitoribosomal subunit

structure. a, Fourier Shell Correlation (FSC) curve of the cryo-EM reconstruction of the $39 \mathrm{~S}$ mitoribosomal subunit. The resolution estimate is $4.9 \AA$ according to the FSC $=0.143$ criterion (gold standard FSC) ${ }^{38} . \mathbf{b}$, Multiparticle refinement of 395 subunit datasets obtained using the FEI Falcon I and Falcon II direct electron detectors. Particle numbers in the classes are indicated below the volumes. Class 1 was used for further refinement of the structure of the 395 subunit to higher resolution. 

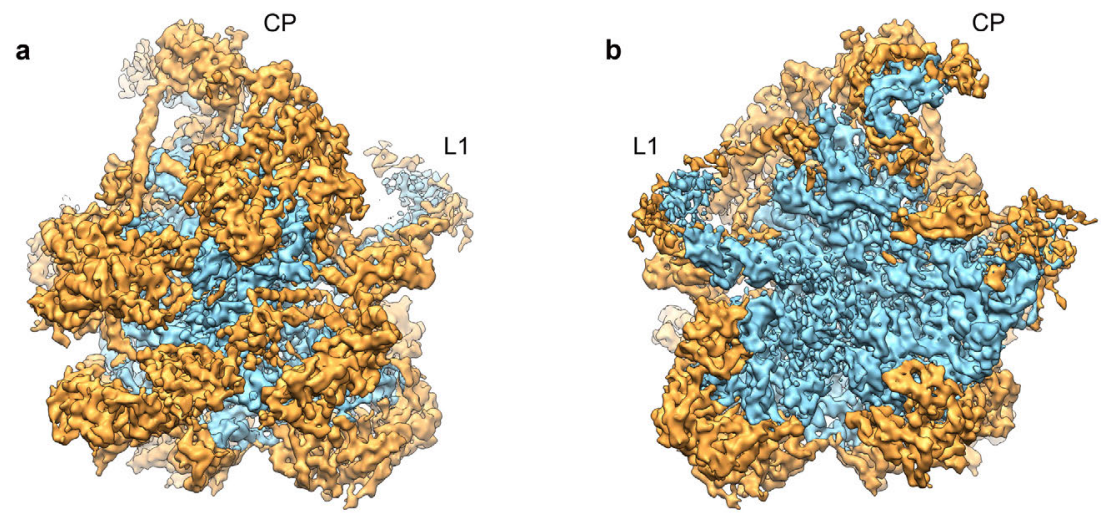

Extended Data Figure 2 | Location of rRNA and proteins in the 395

subunit. $\mathbf{a}$, View from the solvent side and $\mathbf{b}$, from the subunit interface side of the 39S mitoribosomal subunit cryo-EM map, segmented into ribosomal proteins (gold) and rRNA (light blue). (CP, central protuberance; L1, L1 stalk). 


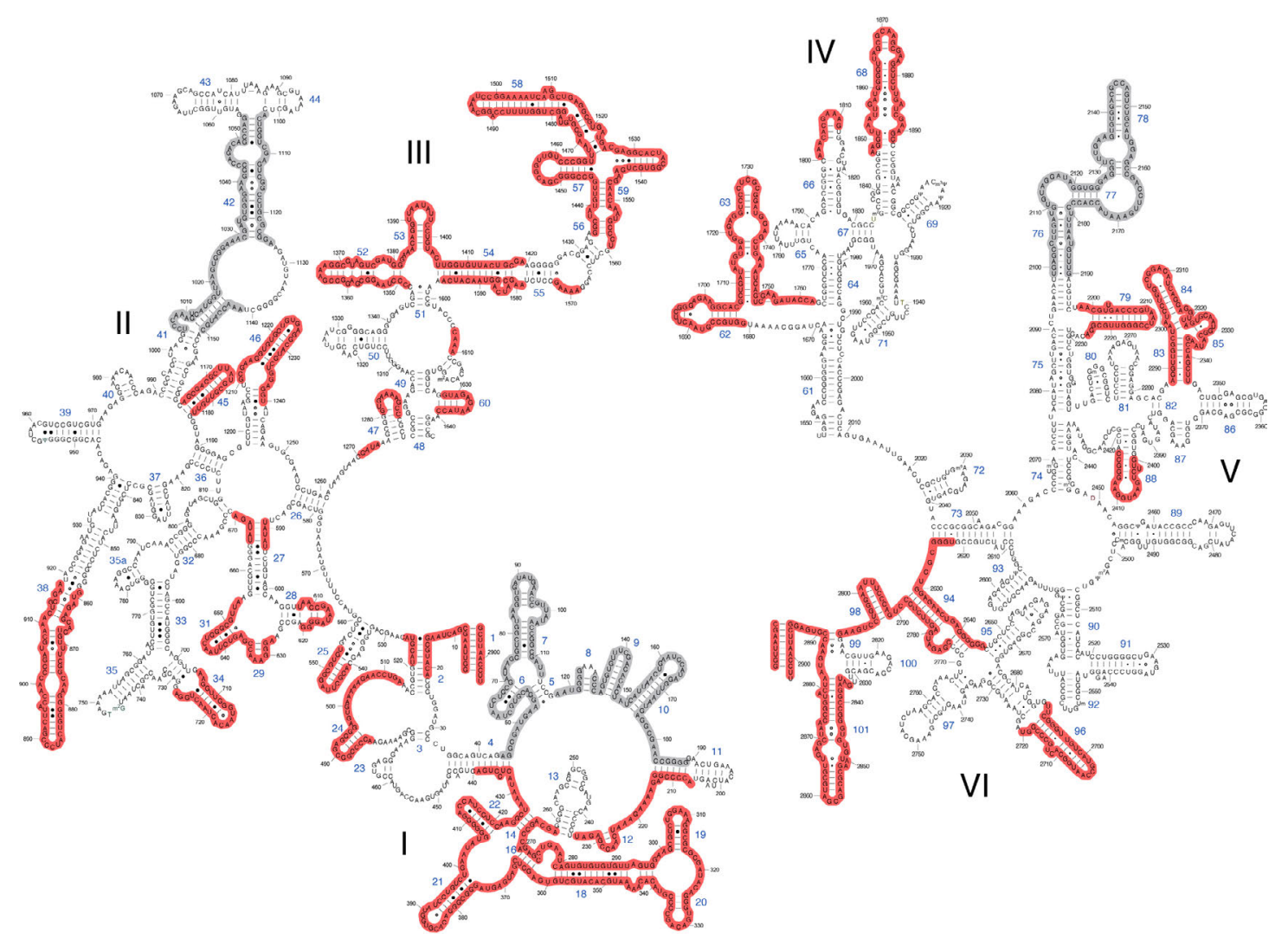

Extended Data Figure 3 | Reductive evolution of the mitochondrial 16S

rRNA. Deletions of rRNA secondary structure elements in mitochondrial $16 \mathrm{~S}$

rRNA are indicated in red on the secondary structure diagram of the

Escherichia coli 23S rRNA. Segments for which the precise locations of

deletions cannot be deduced from our cryo-EM map (the L1 stalk, a part of

domain I rRNA, and the connection to the L7/L12 stalk) are indicated in grey.

Depiction based on the secondary structure of the bacterial $23 S$ rRNA ${ }^{53}$

(template obtained from the Noller lab web page

http://rna.ucsc.edu/rnacenter/noller_lab.html). 


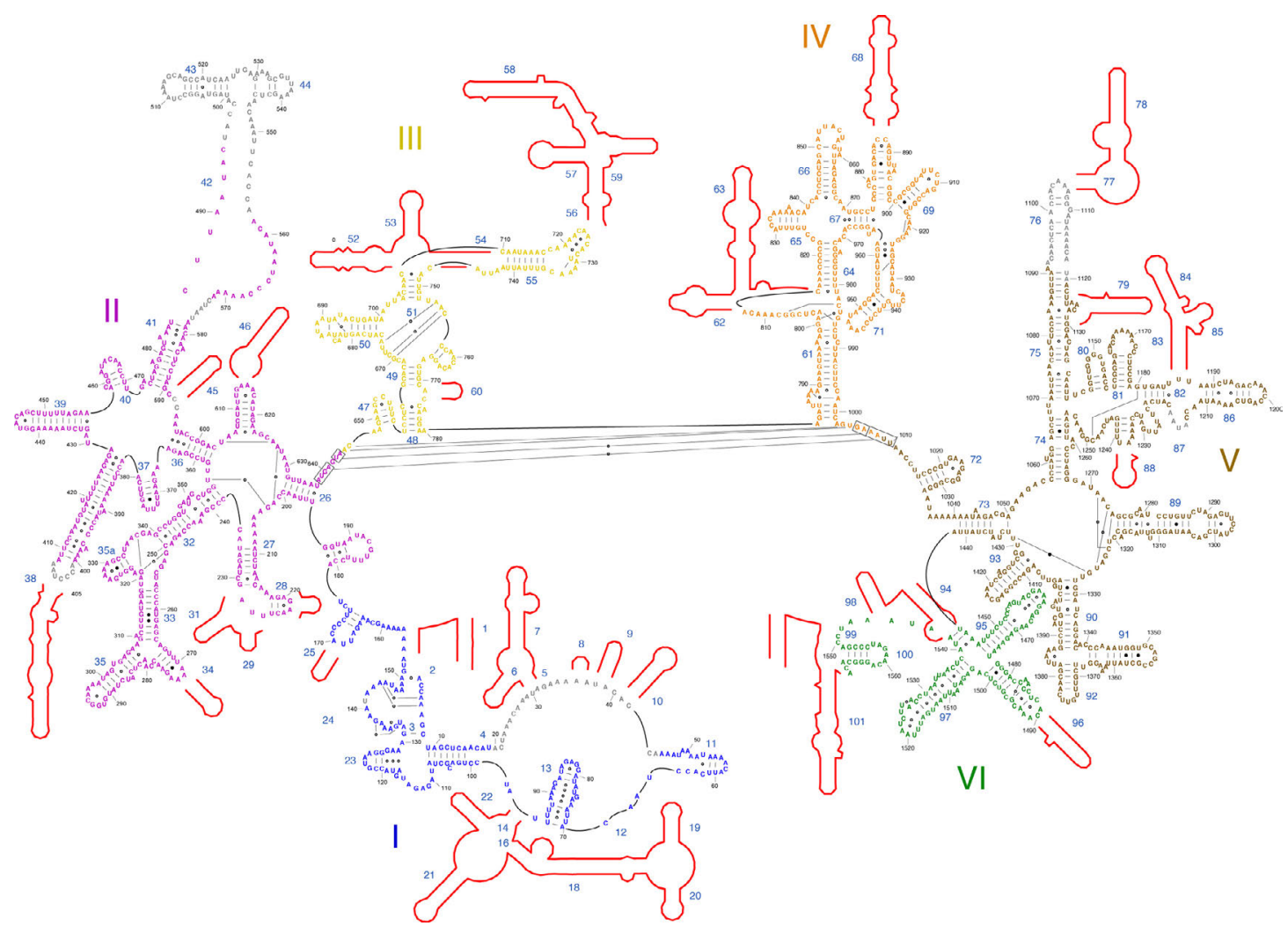

Extended Data Figure 4 | Secondary structure diagram of the S. scrofa large mitoribosomal subunit rRNA. Domains are colored according to Fig. 2 and labeled by Roman numerals. Nucleotides not built in our structure are printed in grey. Bacterial secondary structure elements missing in mammalian mitoribosomes are schematically indicated in red. Watson-Crick base pairs are indicated by lines $(-), G \bullet U$ base pairs by dots $(\bullet)$, and nonstandard base pairs by rings (0). Depiction based on the secondary structure of bacterial $23 \mathrm{~S}$ rRNA ${ }^{53}$ (template obtained from the Noller lab web page http://rna.ucsc.edu/rnacenter/noller_lab.html). 
a

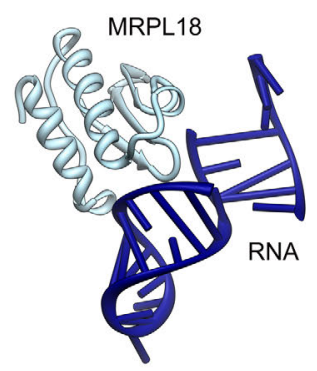

c
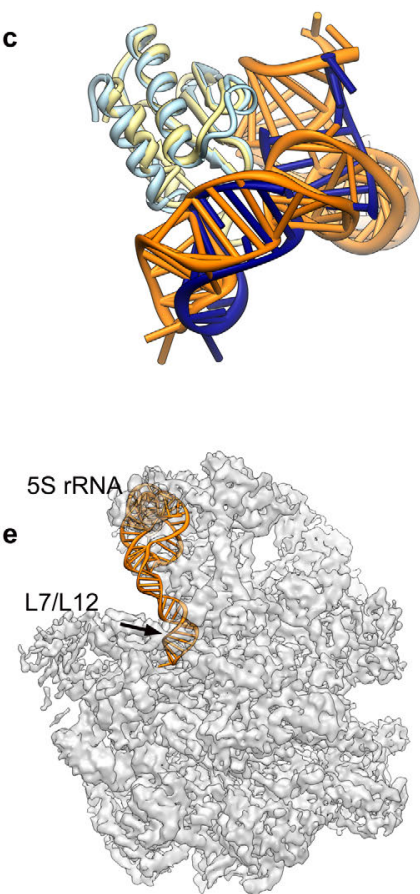

b
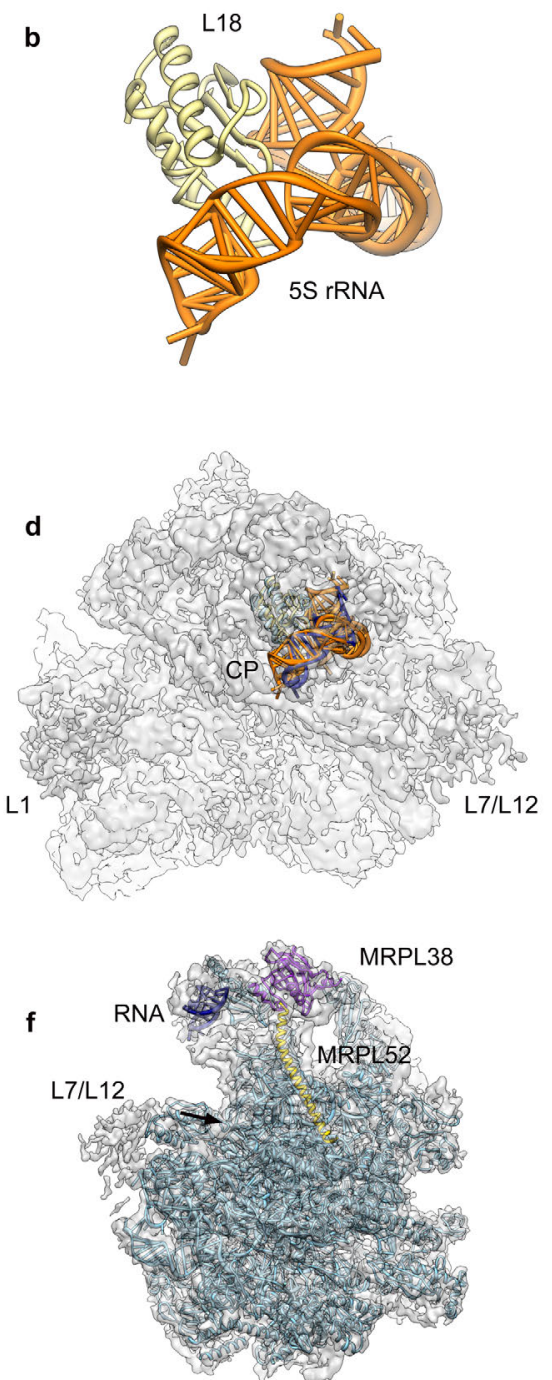

Extended Data Figure 5 | Presence of a second rRNA molecule in the 395 mitoribosomal subunit. a, Depiction of the mitoribosomal CP RNA (dark blue) and the neighboring protein MRPL18 (cyan). b, Depiction of the bacterial 5S rRNA (orange) in complex with ribosomal protein L18 (khaki; PDB ID 3V2D). c, Overlay of $a$ and $b$ according to the homologous proteins L18 and MRPL18. d, Overview of the 39S subunit with the fitted RNAs for orientation. e, In bacterial ribosomes, the $5 \mathrm{~S}$ rRNA connects the CP to the main body of the subunit (the contact region is indicated by an arrow). However, no density for this connection can be seen in the cryo-EM map of the 39S subunit (grey). f, In the mitochondrial 39S subunit, a long a-helical 
protein density corresponding to MRPL52 (gold) instead of the 5 S rRNA connects the mitoribosomal CP (MRPL38, purple) to the subunit body. 

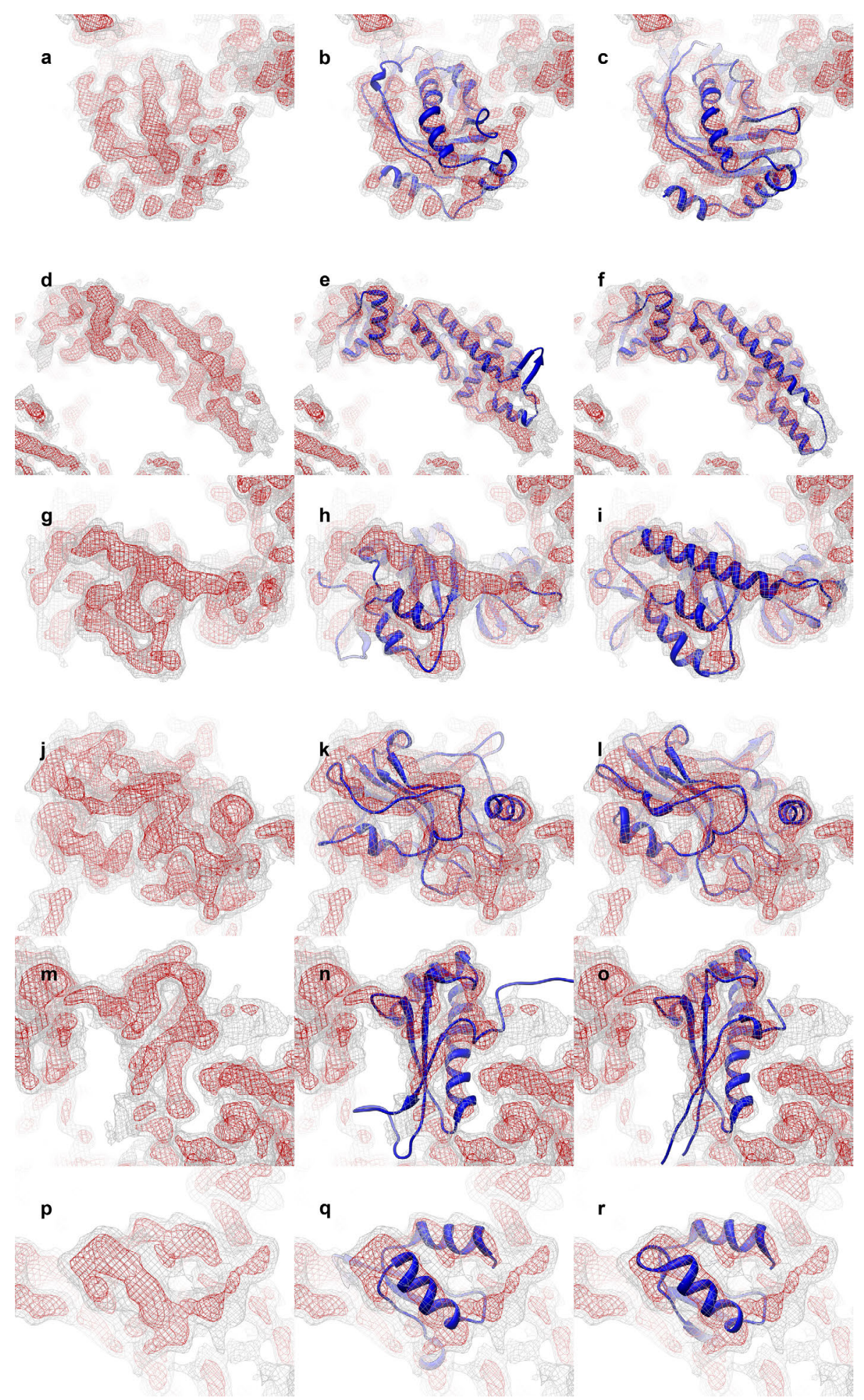

Extended Data Figure 6 | Quality of the fits of newly positioned 39S mitoribosomal proteins in the cryo-EM density. Depiction of the cryo-EM density (first column), fits of the unchanged homology models (second column), and fits of the adjusted final models (third column) of MRPL45 (a-c), 
MRPL44 (d-f), MRPL39 (g-i), MRPL38 (j-l), ICT1 (m-o), and MRPL49 (p-r).

The 39S cryo-EM map is shown at two threshold levels (red and grey, respectively). 


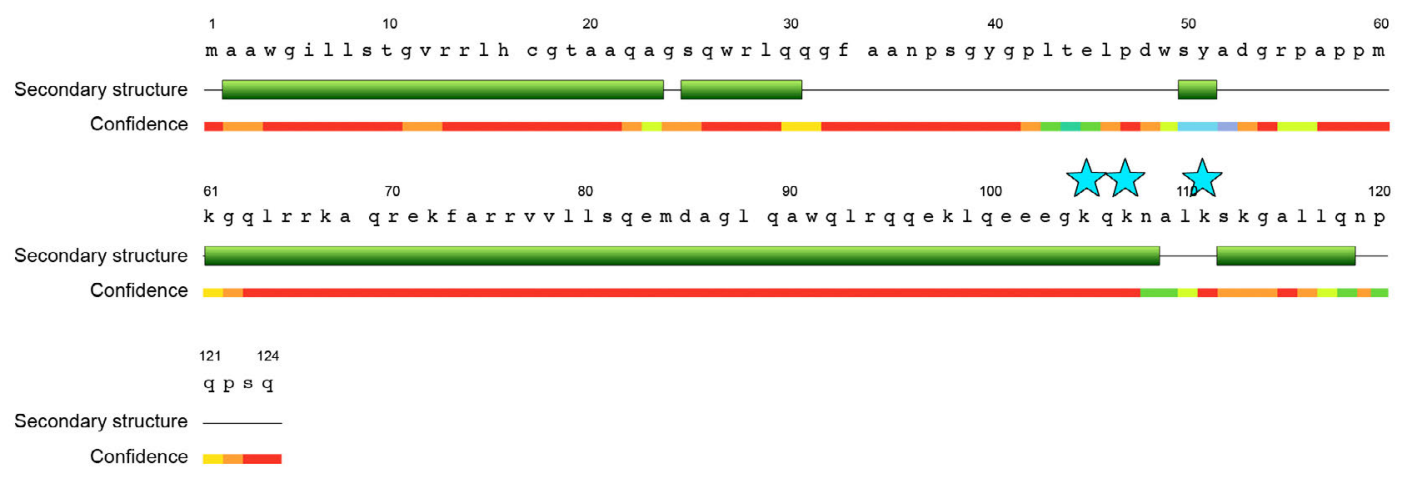

Extended Data Figure 7 | Secondary structure prediction for MRPL52.

The secondary structure prediction (output from Phyre2) ${ }^{19}$ shows a very long (approx. 50 aa) $\alpha$-helix. Blue stars indicate crosslinks of the modeled domain of MRPL38 to MRPL52, as observed in CX-MS experiments. 

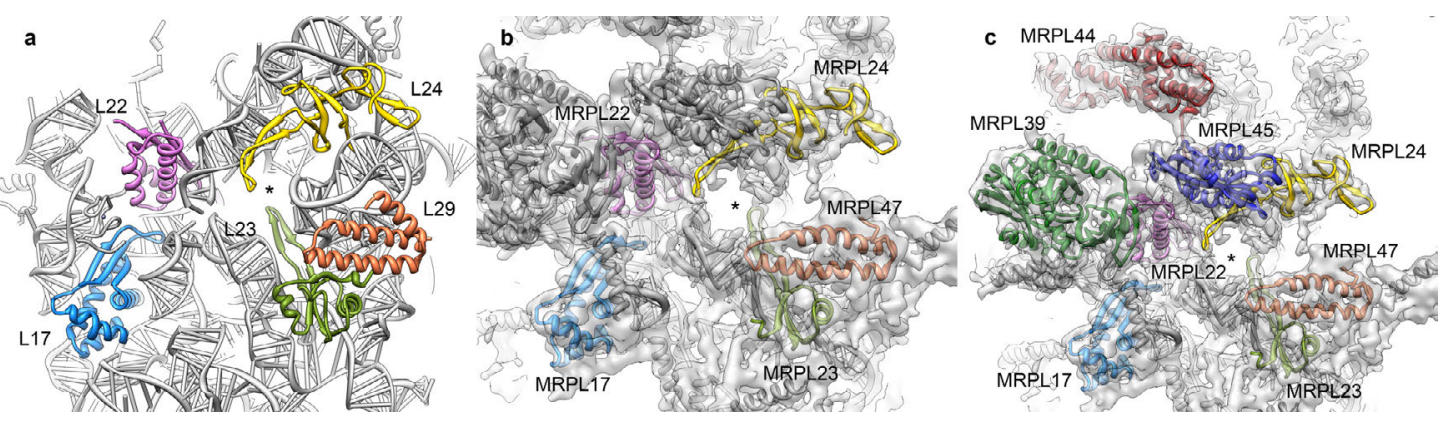

Extended Data Figure 8 | The polypeptide tunnel exit of the

mitoribosomal 395 subunit. a, b, Comparison of the conserved ring of proteins around the polypeptide tunnel exit (indicated by an asterisk). a, The polypeptide tunnel exit in bacteria ${ }^{17}$ (PDB ID 3V2D). b, The polypeptide tunnel exit in the porcine mitoribosome. The ring of proteins around the tunnel exit is conserved. c, Additional mitoribosomal-specific proteins and protein extensions are also located close to the tunnel exit. The newly identified MRPL39, MRPL44 and MRPL45 are shown in green, red, and blue, respectively. 


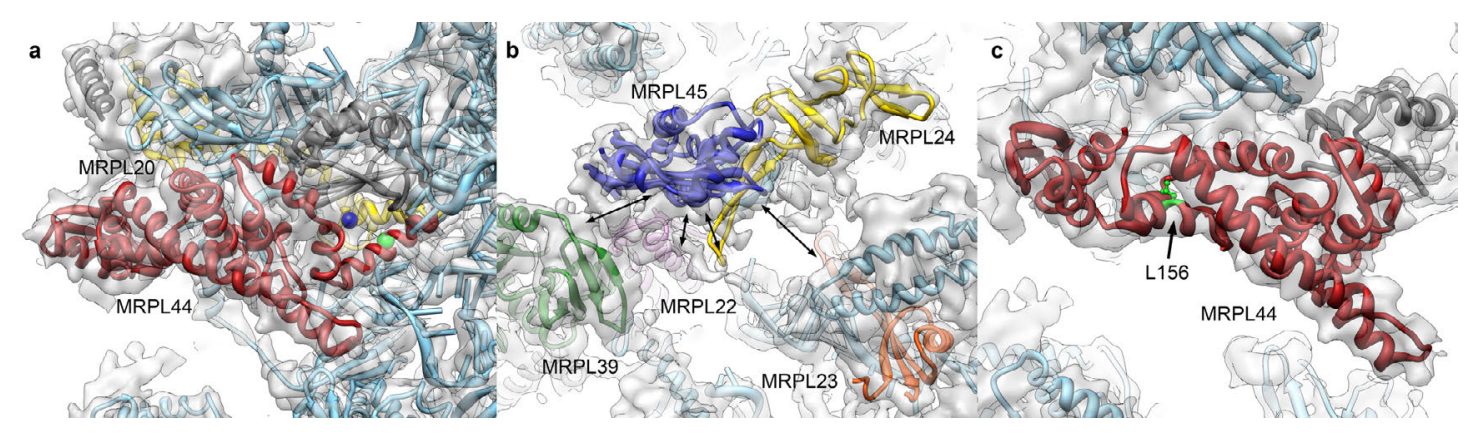

Extended Data Figure 9 | The binding sites of the newly docked mitoribosomal-specific proteins MRPL44 and MRPL45. Crosslinks are indicated by double arrows (see also Supplementary Table 3) unless both crosslinking residues have been modeled in the structure. a, MRPL44 (dark red) has been crosslinked to MRPL20 (gold). The crosslinked sites on MRPL44 and MRPL20 are indicated by green and blue spheres, respectively. b, MRPL45 (dark blue) has been crosslinked to MRPL24 (gold) and MRPL23 (olive) in S. scrofa, as well as MRPL22 (pink) and MRPL39 (green) in B. taurus. For all of these crosslinks, at least one crosslink site is located on mitochondrial-specific extensions that could not be modelled, and therefore these crosslinks cannot be mapped precisely in the structure. c, Mutation in the human homolog of MRPL44 in mitochondrial infantile cardiomyopathy ${ }^{25}$. The L156R mutation (green) maps to a residue not involved in 39S binding. The affected residue is located between two a-helices of the RNase domain, and its mutation likely perturbs the structure of MRPL44 and its capacity to bind the 395 subunit. 
a

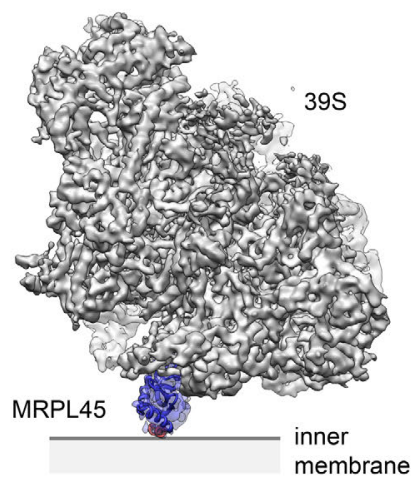

b

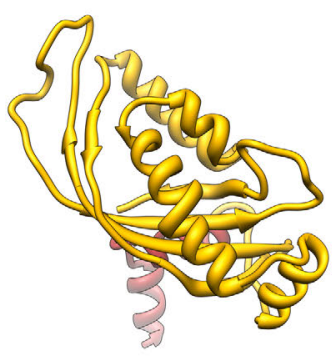

TIM44

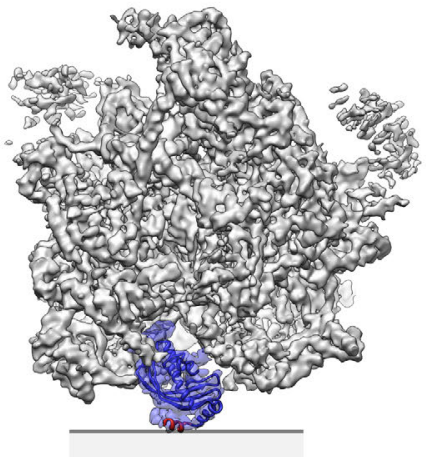

C

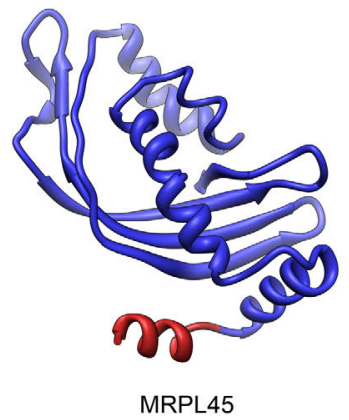

Extended Data Figure 10 | MRPL45 may act as a membrane anchor for the 395 subunit. a, MRPL45 (blue) may anchor the $39 S$ subunit (grey) to the mitochondrial inner membrane. The membrane surface is tentatively indicated by a horizontal line. $\mathbf{b}$, The structure of the C-terminal domain of TIM44 ${ }^{54}$ (PDB ID 2FXT) with its putative membrane interacting segment ${ }^{30}$ is indicated in red. c, The part of MRPL45 corresponding to the putative membrane anchoring segment of TIM44 is shown in red. 